\title{
Neubau-Gentrifizierung und globale Finanzkrise*
}

Der Stadtteil Gallus in Frankfurt am Main zwischen immobilienwirtschaftlichen Verwertungszyklen, stadtpolitischen Aufwertungsstrategien und sozialer Verdrängung

\author{
Sebastian Schipper \\ Felix Wiegand
}

Im Anschluss an die globale Finanzkrise von 2008 lässt sich in vielen deutschen Städten eine neue Welle der Gentrifizierung beobachten. Am Beispiel des traditionellen Arbeiterviertels Gallus in Frankfurt am Main zielt der Beitrag darauf ab, die gegenwärtigen Verdrängungsprozesse aus dem spezifischen Zusammenspiel von stadtpolitischen Aufwertungsbestrebungen und immobilienwirtschaftlichen Verwertungsstrategien zu erklären. Vertreten wird dabei die These, dass die Frankfurter Stadtpolitik, entsprechend ihrer neoliberalen Ausrichtung, im Gallus jahrelang Gentrifizierungsprozesse und die Entstehung einer Ertragslücke zwischen gegenwärtiger und potenzieller Verwertung aktiv gefördert hat - letztere aber erst jetzt im Zuge der Krise an Relevanz gewinnt. Nahezu idealtypisch kann man daher anhand des Gallus zeigen, dass Gentrifizierung wesentlich von lokalen politischen Kräfteverhältnissen abhängt, wobei der Rhythmus jedoch von immobilienwirtschaftlichen Verwertungszyklen diktiert wird.

Ersteinreichung: 18. Dezember 2014; Veröffentlichung online: 16. November 2015

An english abstract can be found at the end of the document.

* Der vorliegende Artikel ist vor dem Hintergrund mehrerer kritischer Stadtspaziergänge durch das Gallus entstanden, welche die Autoren seit Juni 2014 auf Anregung der Friedrich-EbertStiftung durchgeführt haben. Wir bedanken uns bei der Stiftung für die Unterstützung.

\section{Einleitung}

Der Begriff ,Gentrifizierung ' beschreibt stadtteilbezogene Aufwertungsprozesse, bei denen „immobilienwirtschaftliche Strategien der Inwertsetzung und/oder politische Strategien der Aufwertung den Austausch der Bevölkerung für ihren Erfolg voraussetzen" (Holm 2014: 102). Verdrängung, ausgelöst durch steigende Mieten und/oder den Abriss bislang vergleichsweise günstigen Wohnraums, stellt also keinen ungewollten Nebeneffekt dar, sondern ist das Wesen der Gentrifizierung (Lees et al. 2008, Marcuse 1986). Im Anschluss an die globale Wirtschafts- und Finanzkrise von 2008 lässt sich gegenwärtig in vielen Städten in Deutschland und anderswo eine neue Phase von derartigen Aufwertungs- und Verdrängungsprozessen beobachten. Diese kann dabei als kriseninduzierte „global urban strategy“ (Smith 2002) gefasst werden, insofern Investitionen in Wohnimmobilien angesichts einer Überakkumulation von Kapital beziehungsweise eines Mangels an profitablen Anlagesphären nun Aussicht auf vergleichsweise hohe Renditen eröffnen. Überschüssiges, global zirkulierendes Kapital fließt - wie bereits in früheren Phasen kapitalistischer Entwicklung (Harvey 2013) - erneut verstärkt in den vermeintlich sicheren und zugleich profitversprechenden 
Immobiliensektor, wodurch Gentrifizierungsprozesse angetrieben werden (Heeg 2013, Krätke 2014). Um den jeweils konkreten Verlauf solcher Prozesse verstehen zu können, sind neben diesen globalen Tendenzen jedoch ebenso die nationale Regulierung von Finanz- und Wohnungsmärkten, lokale politische Machtverhältnisse sowie stadtteilbezogene Spezifika und Pfadabhängigkeiten zentral (Holm 2013a).

Ausgehend von diesem konzeptionellen Hintergrund richtet unser Beitrag den Blick auf jüngere Gentrifizierungsprozesse im traditionellen Arbeiterviertel Gallus in Frankfurt am Main. Der Stadtteil ist aufgrund seiner innerstädtischen Lage und umfangreicher Neubauprojekte auf ehemaligen Industriebrachen (etwa im so genannten ,Europaviertel', siehe Abb. 5) im Zuge des durch die globale Finanzkrise induzierten Immobilienbooms seit 2010 zu einem neuen Hotspot von Aufwertung und Verdrängung avanciert und jüngst in den Fokus öffentlicher Debatten gerückt. Wie mehrere aus dem Stadtteil entstandene Filmprojekte[1] und von Stadtteilinitiativen durchgeführte Straßenbefragungen veranschaulichen, sind Ängste, perspektivisch aus dem Viertel verdrängt zu werden, weit verbreitet. Diese durchaus berechtigten Befürchtungen drücken sich insbesondere in einer neuen Welle wohnungspolitischer Proteste aus, deren spektakulärste und sichtbarste Spitze in den letzten zwei Jahren insgesamt vier - nach kurzer Zeit von einem Großaufgebot der Polizei geräumte - Hausbesetzungen bilden (Abb. 1).

In auffälligem Kontrast zu alltagsweltlichen Beobachtungen der ortsansässigen Bevölkerung und den zunehmenden wohnungspolitischen Konflikten sind sich stadtpolitische Eliten jedoch uneins, ob die Diagnose Gentrifizierung für das Gallus überhaupt zutrifft. Während etwa der Stadtplanungsamtsleiter Dieter von Lüpke noch 2012 betont, dass ,auch in Zukunft keine ernstzunehmende Gefahr“ bestünde, da das Gallus „nicht aktiv von Gentrifizierung bedroht“ (Frankfurter Rundschau (FR) 15.09.2012) sei, prognostiziert der ehemalige Stadtplanungsdezernent Martin Wentz (SPD) im gleichen Jahr: „In drei Jahren werden wir über die Gentrifizierung des Gallus sprechen“ (Frankfurter Allgemeine Zeitung (FAZ) 29.07.2012). Dort, wo politische Entscheidungsträger_innen den Gentrifizierungsdruck nicht grundsätzlich

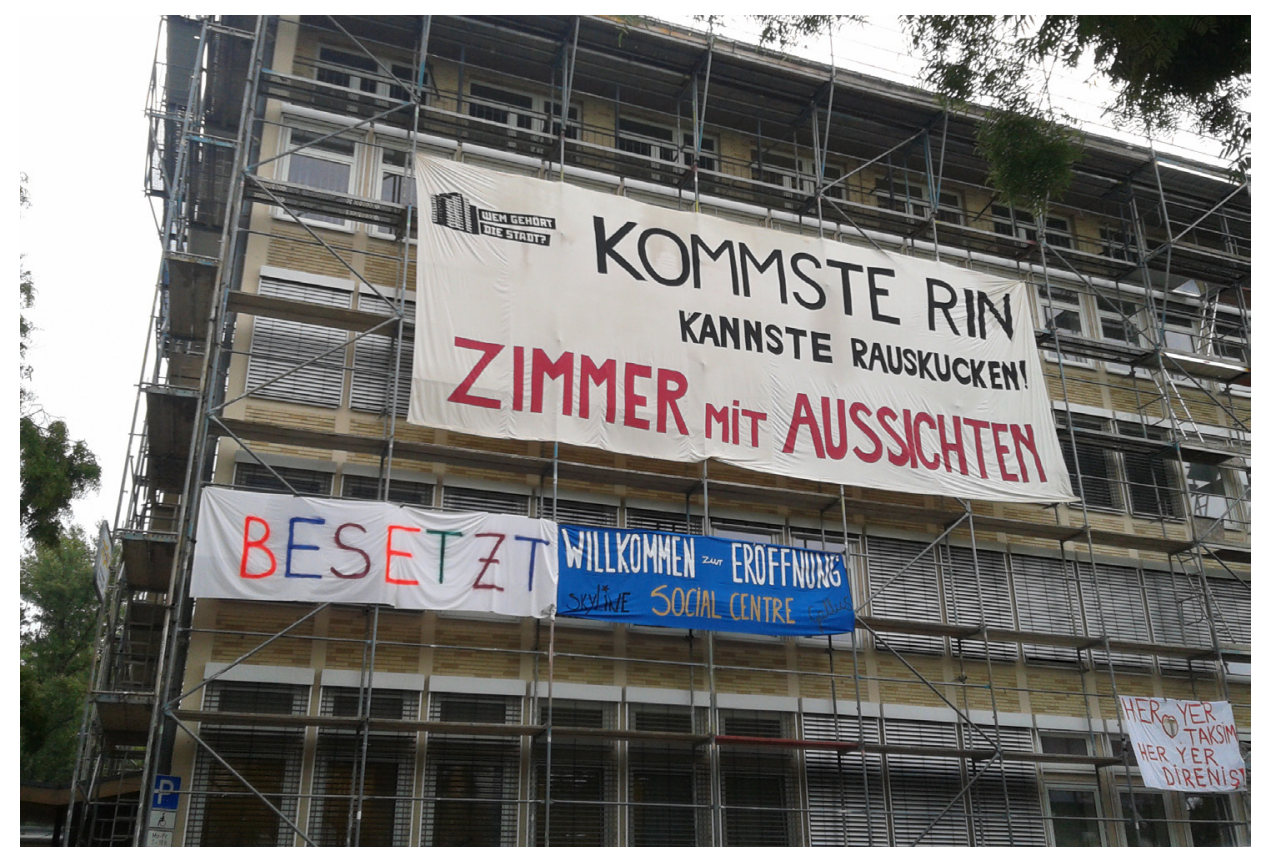

Abb. 1 Besetztes Haus in der Kriftelerstraße im nördlichen Gallus, Sep. 2013 (Foto: Sebastian Schipper) 
bestreiten, wird die Aufwertung häufig als erwünschte Entwicklung aktiv begrüßt und/oder Verdrängung als natürlicher, politisch nicht beeinflussbarer und daher unvermeidbarer Ausdruck einer dynamischen Stadtentwicklung legitimiert (FAZ 29.07.2012, FR 10.07.2013).

In Anbetracht dieses Spannungsverhältnisses zwischen den Positionen der stadtpolitischen Eliten auf der einen Seite und jenen der Bewohnerschaft beziehungsweise stadtpolitischer Aktivist_innen auf der anderen, wollen wir den konkreten Verlauf und die spezifischen Ursachen der Entwicklungen vor Ort näher analysieren. Ziel und Anspruch ist dabei, die gegenwärtigen Verdrängungsprozesse im Gallus als spezifisches Zusammenspiel von stadtpolitischen Aufwertungsstrategien im Kontext neoliberaler Stadtentwicklung (Brenner/Theodore 2002, Schipper 2013a) sowie die immobilienwirtschaftlichen Verwertungsstrategien im Kontext der globalen Finanzkrise zu erklären. Vertreten werden wir erstens die These, dass die Frankfurter Stadtpolitik jahrelang Gentrifizierungsprozesse durch Maßnahmen zur Steigerung der potenziellen Grundrente aktiv gefördert hat, die daraus resultierende Ertragslücke (rent gap) zur bisherigen Verwertung allerdings erst jetzt im Zuge der globalen Finanzkrise ab 2008 an Relevanz gewinnt. Darüber hinaus werden wir zweitens argumentieren, dass diesbezüglich insbesondere die Wirkungsmechanismen einer sogenannten NeubauGentrifizierung (Davidson/Lees 2005) ausschlaggebend sind. In der internationalen Debatte benennt dieser Begriff einen in Deutschland bisher noch relativ selten erforschten Prozess, bei dem gehobener Wohnungsbau zwar direkt keine Verdrängung auslöst, etwa weil selbiger auf ehemaligen Brachflächen errichtet wird, die umfangreichen Investitionsvolumina jedoch aufgrund von Bodenpreissteigerungen und symbolischen Ausstrahlungseffekten Aufwertungs- und Verdrängungsprozesse in der unmittelbaren Nachbarschaft auslösen (Stabrowski 2014). Schließlich werden wir drittens darlegen, dass die Stadt Frankfurt - im Gegensatz zu vielen anderen, finanzschwachen Kommunen - trotz allem weiterhin sehr effektive Instrumente zur Hand hätte (vor allem in Gestalt kommunalen Eigentums), um die Verdrängung einkommensschwacher Haushalte zu verhindern. Nahezu idealtypisch kann man daher am Gallus zeigen, dass Gentrifizierung kein quasi natürliches Resultat rein ökonomischer Marktkräfte ist, sondern wesentlich von lokalen gesellschaftlichen Kräfteverhältnissen abhängt.

Gegliedert ist der Artikel wie folgt: Zuerst werden wir die Wohnungs- und Bodenmarktentwicklungen im Gallus in ihrem gesamtstädtischen Kontext verorten und darlegen, dass das Arbeiterviertel aufgrund einer signifikanten Ertragslücke für Investoren attraktiv geworden ist (Kapitel 2). Um die historische Entwicklung dieser rent gap zu erklären, werden wir in Kapitel 3 den sozio-ökonomischen Wandel des Viertels seit den 1920er Jahren bis in die späten 2000er Jahre rekonstruieren. Dass nun im Anschluss an die globale Finanzkrise von 2008 eine Neubau-Gentrifizierung droht, werden wir in Kapitel 4 aufzeigen. In Kapitel 5 verdeutlichen wir, dass die städtische Wohnungsbaugesellschaft, die Eigentümerin von 30 Prozent des gesamten Wohnraums im Gallus ist, bislang Gentrifizierungsprozesse eher beschleunigt als gebremst hat. Abschließend werden wir in einem knappen Ausblick aktuelle Potenziale für progressive wohnungspolitische Interventionen erörtern. 


\section{Warum ist das Gallus für Investoren attraktiv? Innerstädtische Ertragslücken in Frankfurt am Main}

Zur mikroökonomischen Erklärung von Gentrifizierungsprozessen unterscheidet Neil Smith (1996) zwischen der realen Grundrente, die sich aus den gegenwärtigen Mietzahlungen speist, und der potenziellen Grundrente, die durch die antizipierte profitabelste Verwertung eines Grundstückes beziehungsweise durch die potentiell höchstmöglichen Mietsteigerungen bestimmt ist. Folgt man der Ertragslückentheorie, wird ein Stadtviertel für Immobilieninvestoren insbesondere dann interessant, wenn die Differenz zwischen gegenwärtiger und potentiell durchsetzbarer Grundrente so sehr steigt, dass sich Investitionen angesichts hoher Renditeerwartungen auch im Vergleich zu anderen Anlagesphären lohnen (Clark 1995).

Warum ein solcher Ansatz für die Erklärung der Attraktivität des Gallus relevant ist, lässt sich anhand folgender Karte der innerstädtischen Viertel in Frankfurt veranschaulichen (Abb. 2).[2] Basierend auf dem Mietspiegel von 2012 sind dort die lagespezifischen Zuschläge als Bestandteil der ortsüblichen Vergleichsmiete dargestellt, die wiederum für die Begrenzung von Mieterhöhungen im Bestand ausschlaggebend ist (Schardt 2012). Die lagespezifische Verteilung der $\mathrm{Zu}$ - und Abschläge als Indikator für vergangene Mietpreisentwicklungen zeigt, dass weite Teile der innerstädtischen Viertel mit Hinblick auf die bereits sehr hohe ortsübliche Vergleichsmiete als weitgehend gentrifiziert betrachtet werden können (Bockenheim, Westend, Nordend, Innenstadt, Sachsenhausen, Teilevon Bornheim).[3] Berücksichtigt man zudem, dass viele als einfache Wohnlagen charakterisierte Flächen kaum eine Wohnnutzung zulassen (etwa der neue Universitätscampus im Westend oder der Hafen im Osten), bleiben im Wesentlichen zwei großräumige

Abb. 2 Mietspiegel der Stadt Frankfurt, Stand 2012 (Quelle: Amt für Wohnungswesen 2012)

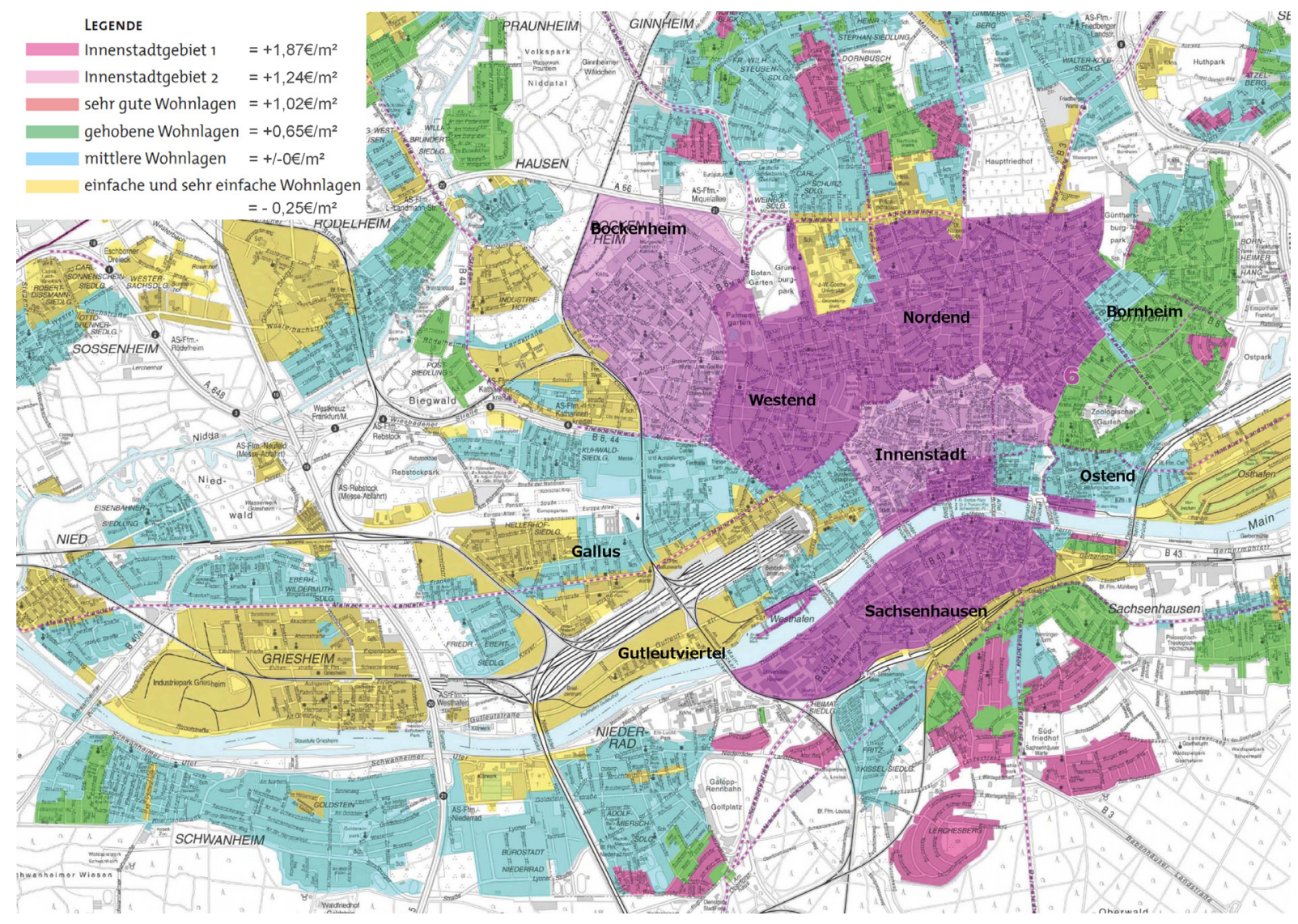


Abb. 3 Entwicklung der Grundrenten in ausgewählten Stadtteilen in Frankfurt am Main (Quelle: Schipper 2013b)

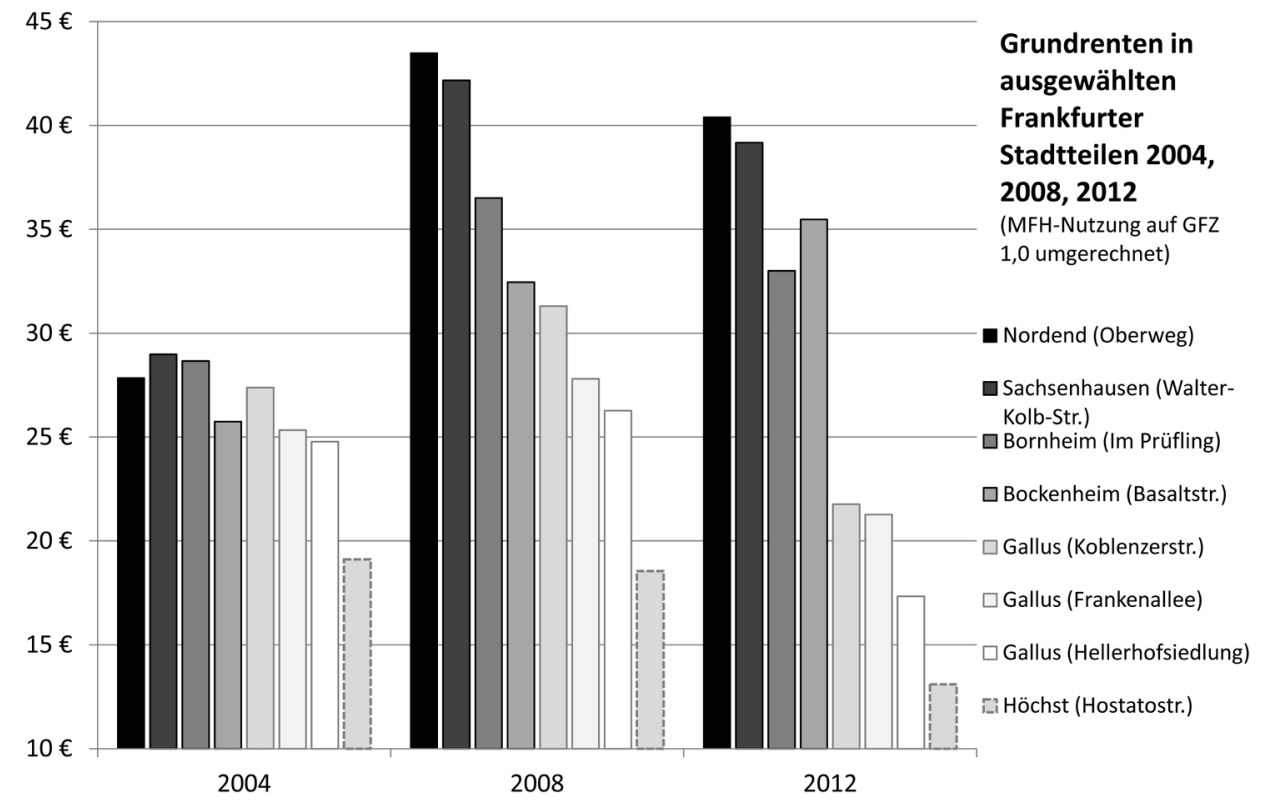

innerstädtische Gebiete, die bislang erschwinglichen Wohnraum in nennenswertem Umfang geboten haben. Dabei handelt es sich zum einen um das Ostend (27.100 EW), das jedoch unter anderem aufgrund des Neubaus der Europäischen Zentralbank unter erheblichem Gentrifizierungsdruck steht, sowie zum anderen um das Gallus (30.200 EW) und das vergleichsweise kleine Gutleutviertel (6.200 EW) westlich der Innenstadt. Angesichts der zentralen Lage dieser Viertel erscheint es aus Sicht von Investoren daher als rational, darauf zu spekulieren, dass sich die Mietentwicklung auch dort zukünftig an derjenigen der anliegenden Viertel orientiert. Insofern Immobilieninvestoren also davon ausgehen, dass eine signifikante Ertragslücke zwischen gegenwärtigen Mietzahlungen und einer höherwertigen Nutzung im gehobenen Wohnsegment existiert und sich diese zukünftig durch entsprechende Strategien der Aufwertung (z. B. Abriss und Neubau, Umwandlung, Sanierung, Mieterwechsel) schließen lässt, steigt der Verwertungsdruck auf die bestehenden Mietverhältnisse und Wohnnutzungen im Gallus.

Abgesehen von den lagespezifischen Unterschieden der ortsüblichen Vergleichsmiete spiegelt sich das Phänomen der Ertragslücke empirisch auch in Bodenpreis- und Grundrentendifferenzen wider. Die bestehenden Grundrentendifferenzen auf Stadtteilebene veranschaulicht exemplarisch Abbildung 3. Dargestellt sind dort die Grundrenten im Gallus im Vergleich zu einer Auswahl von weitgehend gentrifizierten, innerstädtischen Vierteln sowie dem peripheren Stadtteil Höchst. Die Grundrenten wurden aufgrund von Bodenrichtwerten[4] näherungsweise kalkuliert. Es wird erkennbar, dass sich Eigentümer_innen von Grund und Boden im Gallus aufgrund der innerstädtischen Lage schon seit längerem eine im Vergleich zu peripheren Stadtvierteln höhere Grundrente aneignen können, zugleich aber noch eine erhebliche Differenz nach oben zu den bereits gentrifizierten Vierteln existiert. Die vor allem seit 2008 zunehmende Polarisierung des Grundrentenniveaus zwischen den bereits gentrifizierten Vierteln und dem Gallus impliziert, dass die spekulativen Anreize für Eigentümer_innen von Wohnimmobilien, Gentrifizierungsprozesse zu initiieren oder zu befördern, steigen. Denn mit dem Erfolg einer solchen Strategie ließe sich das potenzielle Grundrentenniveau im Gallus auf das ungleich höhere der umliegenden Viertel anheben. 
Dass solche Strategien erfolgreich zu sein scheinen, macht der neu erhobene Mietspiegel von 2014 anschaulich. Abgesehen davon, dass die ortsübliche Vergleichsmiete für ganz Frankfurt im Vergleich zu 2012 im Durchschnitt um 7,2 Prozent (und im Vergleich zu 2010 um 11,3 Prozent) gestiegen ist, erhält nun auch das östliche, innenstadtnahe Gallus als ,erweiterter Innenstadtbereich' erstmalig einen Lagezuschlag von 0,99 Euro/m² (Amt für Wohnungswesen 2014). Die daraus resultierenden Mietsteigerungen können als Indiz interpretiert werden, dass sich die Ertragslücke zwischen realer und potenzieller Verwertung sukzessive schließt - mit dem Effekt, dass einkommensschwache Haushalte aus dem Stadtteil verdrängt werden.[5]

Zusammengefasst kann also die mikroökonomische Ertragslücke als Motor der gegenwärtigen Gentrifizierungsprozesse im Gallus interpretiert werden. Damit ist aber noch nichts darüber gesagt, wie eine solche Lücke historisch entstehen konnte. Vielmehr gilt es zu erklären, warum Mieten und Grundrenten in einem innerstädtischen Viertel in Frankfurt trotz der zentralen Lage lange Zeit vergleichsweise niedrig waren und warum Immobilieninvestoren gegenwärtig offenbar erfolgreich darauf spekulieren, dass Mieten und Wohnungspreise steigen.

\section{Das Gallus - Historischer Abriss}

\subsection{Entstehung eines klassischen Arbeiter_innenviertels}

Das Gallus - mit gegenwärtig 30.200 Einwohner_innen der drittbevölkerungsreichste Stadtteil Frankfurts (Stand 2013) - verdankt seine Entstehung den Industrialisierungs- und Urbanisierungsprozessen des späten 19. Jahrhunderts (Becker 1986, Lauer-Seidelmann 2012). Den historischen Kern des Stadtteils bildet ein Areal im Bereich der damaligen südwestlichen Außenstadt von Frankfurt. Dieses wurde von der Stadt im Zusammenhang mit der in den 1880er Jahren erfolgten Errichtung des Hauptbahnhofes, des Hauptgüterbahnhofes sowie des Westhafens als Fabrikviertel ausgewiesen und infrastrukturell erschlossen. Infolge der raschen Ansiedlung zahlreicher Industrie-, Gewerbe- und Handwerksbetriebe begannen um die Jahrhundertwende verschiedene gemeinnützige Wohnungsbaugesellschaften, in der Nähe der Arbeitsstätten Wohngebäude für Arbeiter_innen sowie einfache Beamte und Angestellte zu errichten. Im Rahmen des kommunalen Wohnungsbauprogrammes Neues Frankfurt[6] wurde diese Bautätigkeit in den späten 1920er und frühen der 1930er Jahren in Gestalt der Hellerhofsiedlung (Abb. 5 und 12) sowie der Friedrich-Ebert-Siedlung noch einmal intensiviert. Diese Projekte repräsentieren zugleich auch den Abschluss der Entwicklung des Gallus zu einem „,klassische[n] $]^{6}$ Arbeiterviertel, in dem, trotz funktionaler Trennung, Arbeiten und Wohnen der Niedrigeinkommenshaushalte auf relativ engem Raum integriert waren“ (Tharun/Unterwerner 1993: 308). Ein wichtiges Moment dieser Prägung war die „lokale Hegemonie der verschiedenen Arbeiterinstitutionen“ (Ronneberger/Keil 1995: 327), also Parteien, Gewerkschaften, Vereine etc., die das ,Rote Gallus' in der Zwischenkriegszeit zu einer Hochburg von SPD und KPD sowie zu einem Ort häufiger Streiks und eines vergleichsweise aktiven Widerstandes gegen den Nationalsozialismus machte (Becker 1986: 51 ff., Lauer-Seidelmann 2012). 


\subsection{Deindustrialisierung und sozio-ökonomischer Niedergang}

Nach dem Zweiten Weltkrieg änderte sich an dem Charakter des Gallus als Industrie- und Arbeiterviertel zunächst wenig. Bis zum Jahr 1961 erreichte die Bevölkerungszahl mit über 37.000 beinahe wieder das Vorkriegsniveau von knapp 40.00o. Im Verlauf der 1960er Jahre und verstärkt seit den $1970 e r$ Jahren setzte dann jedoch eine Reihe umfassender sozio-ökonomischer Restrukturierungsprozesse ein (Kirst 2006, Ronneberger/Keil 1995, Tharun/ Unterwerner 1993, Welz 1992). Insofern diese Veränderungen im breiteren Kontext der Krise des Fordismus beziehungsweise des Übergangs zu einer post-fordistischen, neoliberalen Entwicklungsweise stehen, lassen sich im Gallus die Auswirkungen und Widersprüche dieser gesamtgesellschaftlichen Transformation in räumlich und sozial verdichteter Form beobachten.

Bei diesen Prozessen handelt es sich erstens um den grundlegenden Wandel der ökonomischen Basis des Viertels. Ausschlaggebend hierfür war zunächst eine - sich in mehreren Wellen vollziehende - Restrukturierung und schließlich Abwanderung des produzierenden Gewerbes und eine dadurch ausgelöste großflächige Deindustrialisierung. So wurden im Gallus von 1970 bis 1987 annähernd 6o Prozent der Arbeitsplätze im produzierenden Gewerbe abgebaut, ohne dass diese negative Beschäftigungsentwicklung durch das parallel einsetzende Wachstum des Dienstleistungssektors hätte aufgefangen werden können (Tharun/Unterwerner 1993: 306). Dass Deindustrialisierung und Tertiarisierung insbesondere in den 1980er Jahren an Dynamik gewannen, lag dabei nicht zuletzt an der (Planungs-)Politik der Stadt Frankfurt, die diese Restrukturierungsprozesse - ihrer generell zunehmend unternehmerischen, konkurrenzorientierten Ausrichtung (Schipper 2013a) folgend - „von Anfang an [...] weitgehend wohlwollend und flexibel auf Investorenwünsche reagierend [begleitet hat]“ (Tharun/ Unterwerner 1993: 311, vgl. auch Kirst 2006: 40 ff.). Charakteristisch hierfür war unter anderem der so genannte ,Speerplan' (1983), der die quer durchs Gallus verlaufende Mainzer Landstraße (insbesondere den innenstadtnahen östlichen Teil bis zur Galluswarte) als eine der zentralen Achsen der geplanten City-Erweiterung auswies und eine entsprechend hochverdichtete Arbeitsplatznutzung in Bürohochhäusern vorsah. Als Reaktion auf diese Planung und angesichts eines generellen, spekulativen Immobilienbooms im Kontext der Global City-Formierung Frankfurts (Schipper 2013b: 191 ff.) nahmen Immobilieninvestoren ab Mitte der 1980er Jahre eine positive Neubewertung des Gallus vor, dessen Lagepotential zuvor wegen der sozioökonomischen Struktur sowie des Standortes zwischen den Gleisanlagen des Güterbahnhofes im Norden und des Hauptbahnhofes im Süden eher gering eingeschätzt worden war. Infolgedessen stiegen die Bodenpreise im östlichen Teil der Mainzer Landstraße im Verlauf der 1980er Jahre um das Zehnfache, die Mehrzahl der Grundstücke wechselte den_die Besitzer_in (Tharun/ Unterwerner 1993: 306f). Wie etwa die im Jahr 1992 unter starkem Protest vollzogene Schließung der traditionsreichen Büromaschinenfabrik TriumphAdler exemplarisch verdeutlicht, wurde es unter diesen Bedingungen selbst für Industrieunternehmen und Gewerbebetriebe westlich der Galluswarte zunehmend lukrativer, den Grundstückwert ihrer Betriebsimmobilie zu realisieren, statt die Produktion weiterzuführen. 
Parallel und in enger Verbindung mit diesen ökonomischen Veränderungen verlief zweitens eine tiefgreifende soziale Transformation des Viertels. Diese beruhte zunächst auf einer bereits in den 1960er Jahren einsetzenden - und keineswegs auf das Gallus beschränkten - Abwanderung der besserverdienenden, mehrheitlich deutschen (Fach-)Arbeiter_innen, Beamten und Angestellten, die aus den überwiegend kleinen und renovierungsbedürftigen Wohnungen[7] in Neubaugebiete am Stadtrand zogen. So verlor das Gallus zwischen 1970 und 198742 Prozent seiner deutschen Wohnbevölkerung (Tharun/Unterwerner 1993: 309). Ersetzt wurde sie durch,Gastarbeiter_innen', die aufgrund vielfältiger rassistischer Diskriminierungen nur zum unteren Segment des Wohnungsmarktes Zugang hatten und wegen der im städtischen Vergleich eher geringen - und trotzdem häufig überteuerten - Mieten ins Gallus zogen (Borris 1973: 129 ff.). In Folge stieg der Migrant_innen-Anteil an der Gesamtbevölkerung kontinuierlich auf gegenwärtig knapp 42 Prozent. Die mit dieser Entwicklung verbundene Entstehung zahlreicher migrantischer Vereine, Netzwerke und Gewerbe, aber auch die strukturelle Benachteiligung des Stadtviertels, unter anderem infolge der markanten Unterrepräsentation bei Wahlen, prägen das Gallus bis heute nachhaltig. Der starke Zuzug von Arbeitsmigrant_innen konnte jedoch nicht verhindern, dass die Bevölkerung des Viertels zwischen 1970 und 1987 um insgesamt 22 Prozent zurückging (Tharun/Unterwerner 1993: 309). Da im selben Zeitraum infolge der umfangreichen Deindustrialisierung die Zahl der Arbeitslosen und Sozialhilfeempfänger_innen deutlich anstieg und die traditionellen proletarischen Netzwerke und Institutionen erodierten, erhielt das Gallus im städtischen Diskurs ein deutlich negatives, häufig klassistisch und rassistisch konnotiertes Image. In diesem Sinne diagnostizierten Anfang der 1990er Jahre, als es nach der temporären Zunahme des Zuzugs von Migrant_innen zur Überbelegung einzelner Wohnungen kam, nicht mehr nur die Boulevardmedien, sondern auch kritischeStadtforscher_innen eine „beginnende Ghettobildung“(Ronneberger/Keil 1995: 327).

\subsection{Gescheiterte Gentrifizierungsversuche und der Status quo}

Parallel zu solchen Diagnosen des Niedergangs wurden Ende der 1980er und zu Beginn der 1990er Jahre im Kontext der Tertiarisierungsprozesse und spekulativer Immobiliengeschäfte im Gallus indes auch erstmals Warnungen vor einem steigenden Aufwertungsdruck, der Umwandlung von billigem Wohnraum in Büroflächen sowie der Verdrängung der angestammten, einkommensschwachen Bevölkerung laut. Statt gegen einzelne Studierende und Kulturschaffende, die seit Mitte der 1970er Jahre bestenfalls „sporadisch zu Pionieren einer kleinräumigen Gentrifzierung“ (Tharun/ Unterwerner 1993: 309) geworden waren, richtete sich die Kritik mehrheitlich gegen die investorenfreundliche Politik der Stadt, die es zum Beispiel unterlassen hatte, Investoren mittels baurechtlicher Verordnungen zur Errichtung bezahlbaren Wohnraumes zu verpflichten oder die Umwandlung von Wohn- in Büroraum zu verhindern (ebd.: 311 f., Welz 1992).

Trotz eines kontinuierlichen Anstiegs des generellen Mietniveaus - gerade auch in den Wohnsiedlungen der städtischen Wohnungsbaugesellschaft ABG (Kirst 2006: 87) - erwiesen sich Befürchtungen vor einer großflächigen 
„Yuppiefizierung“ (Welz 1992: 85) des Gallus jedoch insofern als verfrüht, als eine Mitte der 1990er Jahre einsetzende Immobilienkrise (Schipper 2013b: 192) die Dynamik der sozialräumlichen Transformation des Gallus deutlich abbremste. In der Folge wurden viele geplante Umwandlungs- und Neubauprojekte temporär gestoppt, so dass umfassender Leerstand und mehrere, vor allem im Westen des Gallus gelegene Industrie- und Gewerbebrachen einen nun zunehmend „schleppende[n] Strukturwandel“ (Kirst 2006: 84) beziehungsweise das Scheitern der städtischen Aufwertungspolitik anzeigten. Auch wenn die 1998 erfolgte Aufgabe des Hauptgüterbahnhofes und die anlaufende Planung für das Europaviertel sowie ein weiterer Immobilienboom um die Jahrtausendwende erneut als Zeichen einer beginnenden Aufwertung gedeutet wurden, änderte sich am Status quo zunächst wenig. Während die Bevölkerungszahl im Verlauf der 1990er Jahre weitgehend stagnierte, nahm insbesondere die Dichte an Sozialhilfeempfänger_innen weiter zu, was dem Gallus endgültig den Status als „neues Armutsgebiet“ einbrachte (Klagge 2005: 182 f.). Als Reaktion auf die räumliche Konzentration sozial benachteiligter Bevölkerungsgruppen und einer auch städtebaulich schwierigen Situation (z. B. starke Umwelt- und Verkehrsbelastung, Mangel an öffentlichen Grün- und Spielflächen), wurde das Viertel im Jahr 2001 in das Bund-Länder-Programm Soziale Stadt für ,Stadtteile mit besonderem Entwicklungsbedarf' aufgenommen (Stadt Frankfurt 2006). Neben der Einführung neuartiger Steuerungs- und Beteiligungsverfahren (u. a. Quartiersmanagement, Stadtteilbüro) dienten die Fördermittel in Höhe von knapp 10 Millionen Euro - sowie weitere 20 Millionen Euro aus städtischen Programmen - seither vor allem dazu, verschiedene soziale, insbesondere (aus-)bildungs- und beschäftigungsorientierte Projekte anzustoßen sowie städtebauliche Maßnahmen durchzuführen (z. B. der Bau eines Quartierspavillons, die Sanierung und Umgestaltung öffentlicher Plätze oder die Errichtung von Fuß- und Radwegen, Spielplätzen etc.). Im Rahmen einer umfassenden Öffentlichkeitsarbeit wurde zudem, etwa in Zusammenarbeit mit dem örtlichen Gewerbeverein, aktiv versucht, das Image des Stadtviertels zum Positiven zu verändern, wozu unter anderem auch die Schließung beziehungsweise Umgestaltung mehrerer, Wasserhäuschen ' (Trinkhallen bzw. Kioske) beitragen soll.

Das in diesem Zusammenhang gezeichnete Bild, wonach das Gallus in

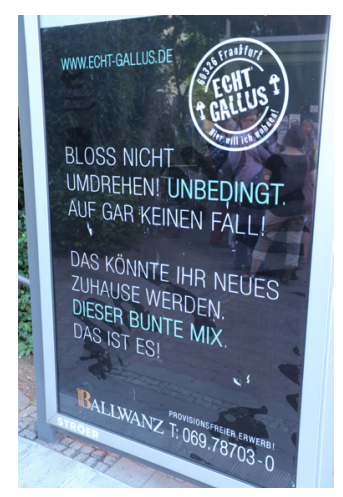

Abb. 4 Werbung für Eigentumswohnungen in der Kleyerstraße, Aug. 2014 (Foto: David Schommer) den letzten Jahren einen , positiven Wandel' erfahren habe, hat in der öffentlichen Wahrnehmung mittlerweile den lange dominierenden Diskurs über ein Viertel im sozio-ökonomischen Niedergang größtenteils abgelöst. Wie weit diese Verschiebung inzwischen reicht, veranschaulichen immobilienwirtschaftliche Werbestrategien, in denen etwa unter dem Slogan „Echt Gallus“ die zuvor lange Zeit negativ konnotierte soziale und ethnische Vielfalt des Viertels - sein ,bunter Mix - als besondere Standortqualität hervorgehoben wird (Abb. 4). Dieser Imagewandel darf indes nicht darüber hinwegtäuschen, dass das Gallus heute zwar zurecht als „sozial stabil“ (Kirst 2006: 92) gilt, es aber nach wie vor ein Stadtviertel ist, in dem einkommens- und sozial schwache Haushalte überrepräsentiert sind: So liegt insbesondere die Arbeitslosenquote (7 Prozent gegenüber 5,3 Prozent) sowie der Anteil von Empfänger_innen staatlicher Sozialleistungen (20 Prozent gegenüber 12,6 Prozent) jeweils deutlich über dem städtischen Durchschnitt 
(Bürgeramt 2013: 80, 126), während sich umgekehrt der Einkommensmedian (brutto, sozialversicherungspflichtig, Vollzeit) mit 2.620 Euro gegenüber dem gesamtstädtischen Mittel von 3.291 Euro auf vergleichsweise niedrigem Niveau befindet (Bürgeramt 2012: 84).

Im Anschluss an die globale Wirtschafts- und Finanzkrise von 2008 ist das Gallus jedoch erneut in den Fokus immobilienwirtschaftlicher Verwertungsstrategien gerückt. Angesichts eines krisenbedingten Mangels an profitablen Anlagesphären einerseits und der mikroökonomischen Ertragslücke im Gallus andererseits versprechen Wohnimmobilien hier plötzlich vergleichsweise hohe Renditen. Die in den 1990er Jahren noch gescheiterten Aufwertungsprozesse und Großprojekte haben dadurch wieder an Dynamikgewonnen. Um zu veranschaulichen, wie auf ehemaligen Brachflächen (Abb. 5) ein überwiegend gehobenes Wohnangebot realisiert und dadurch Prozesse der NeubauGentrifizierung (Davidson/Lees 2005) ermöglicht worden sind, werden wir im Folgenden am Beispiel des Europaviertels und anderer Konversionsflächen das Zusammenspiel aus immobilienwirtschaftlichen Verwertungsstrategien und stadtpolitischer Rahmensetzung näher beleuchten.

\section{Neubau-Gentrifizierung im Kontext stadtpolitischer Aufwertungsstrategien: Das Europaviertel und andere Konversionsflächen im Gallus}

\subsection{Das neue Gallus: Wohnen im Europaviertel}

Mit rund 145 Hektar Fläche und einer Investitionssumme von mehreren Milliarden Euro ist das in nördlicher Nachbarschaft zum Gallus gelegene (und administrativ diesem zugehörige) Europaviertel eines der zurzeit umfassendsten Stadtentwicklungsprojekte Europas. Nach seiner Fertigstellung Anfang der 2020er Jahre soll es, so die offiziellen Angaben, neben 30.000 Arbeitsplätzen und Erweiterungsflächen für die Messe auch bis zu 6.00o Wohnungen bieten. Seinen Ausgang nahm das Projekt Mitte der 1990er Jahre, als die Deutsche Bahn AG die Aufgabe des Hauptgüterbahnhofs bekannt gab. Neben dem Rückgang des Transportvolumens im Güterverkehr lässt sich diese Entscheidung insbesondere auf das gesteigerte Interesse der Bahn an einer möglichst profitablen Verwertung ihrer größtenteils innenstadtnahen Liegenschaften zurückführen (Langhagen-Rohrbach 2003: 147 f).[8]

Als „Startschuss“ (Langhagen-Rohrbach 2003: 148) für das Europaviertel diente eine bereits vor der 1998 erfolgten Betriebsaufgabe des Güterbahnhofes veröffentliche Absichtserklärung, in der eine der beiden neuen Grundstückseigentümerinnen[9], die Stadt Frankfurt sowie die Firma Stella AG den Bau eines mehr als eine Milliarde DM teuren Urban Entertainment Centers am östlichen Geländeeingang ankündigten. Dieses Vorgehen ist charakteristisch für den gesamten Planungsprozess, in dem die Eckpunkte des Projektes durch Absprachen einflussreicher Akteur_innen vorab festgelegt wurden und die Stadt eine profitorientierte Entwicklung durch das Aufstellen eines großzügigen Hochhausentwicklungsplanes sowie den weitgehenden Verzicht auf sozialpolitische Vorgaben aktiv beförderte. Zudem wurde - zumindest zu Beginn - über zentrale Planungsschritte, wie z. B. die Erstellung des Rahmenplanes, „hinter verschlossenen Türen“ 
Abb. 5 Neubauprojekte im Gallus und Europaviertel, Stand Sept. 2014 (Karte: Elke Alban)

Abb. 6 Blick von der Emser Brücke auf das östliche Europaviertel und die Europa-Allee, Juni 2013 (Foto: CC Epizentrum)

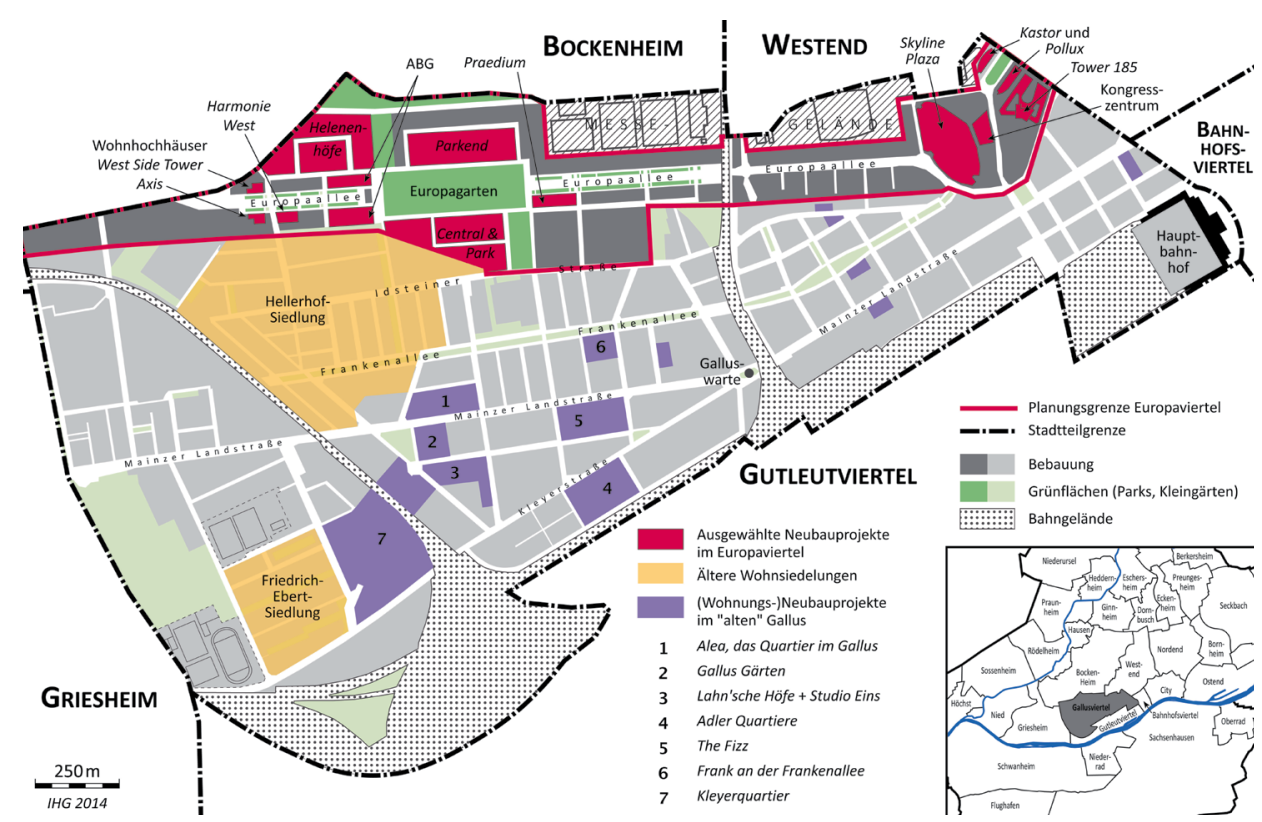

(ebd.: 149), das heißt ohne Beteiligung einer kritischen Öffentlichkeit entschieden. Auch deshalb blieben frühzeitige Warnungen einer Bürgerinitiative und von kritischen Stadtforscher_innen, dass vom Europaviertel „eine Sogwirkung auf das benachbarte Gallus ausgehen“ werde, weitgehend ungehört: „Die dortigen Mietpreise werden steigen, Wohnungen werden luxussaniert werden, Leute mit wenig oder ohne Einkommen strukturell verdrängt.“ (WobiG 1998, vgl. auch Kirst 2006, Langhagen-Rohrbach 2003: 156 f.).

Betrachtet man die konkreten Projekte, die im Europaviertel entlang der in Ost-West-Richtung verlaufenden Europa-Allee realisiert worden sind (Abb. 5), wird ersichtlich, warum diese Befürchtungen mehr als berechtigt sind. So entstanden seit der zweiten Hälfte der 2000er Jahre im östlich der Emser Brücke gelegenen Areal neben der bereits 2001 abgeschlossenen Erweiterung der Frankfurter Messe mehrere Hotels, Geschäfts- und Büroflächen sowie Gebäudekomplexe mit hochpreisigen Miet-und Eigentumswohnungen in geschlossener Blockrandbebauung (Abb. 6). Zudem wurden hier anstelle des zunächst geplanten Urban Entertainment Centers - und unter Verzicht auf den hierfür ursprünglich vorgesehenen Wohnanteil von 30 Prozent - das Einkaufszentrum Skyline Plaza sowie ein

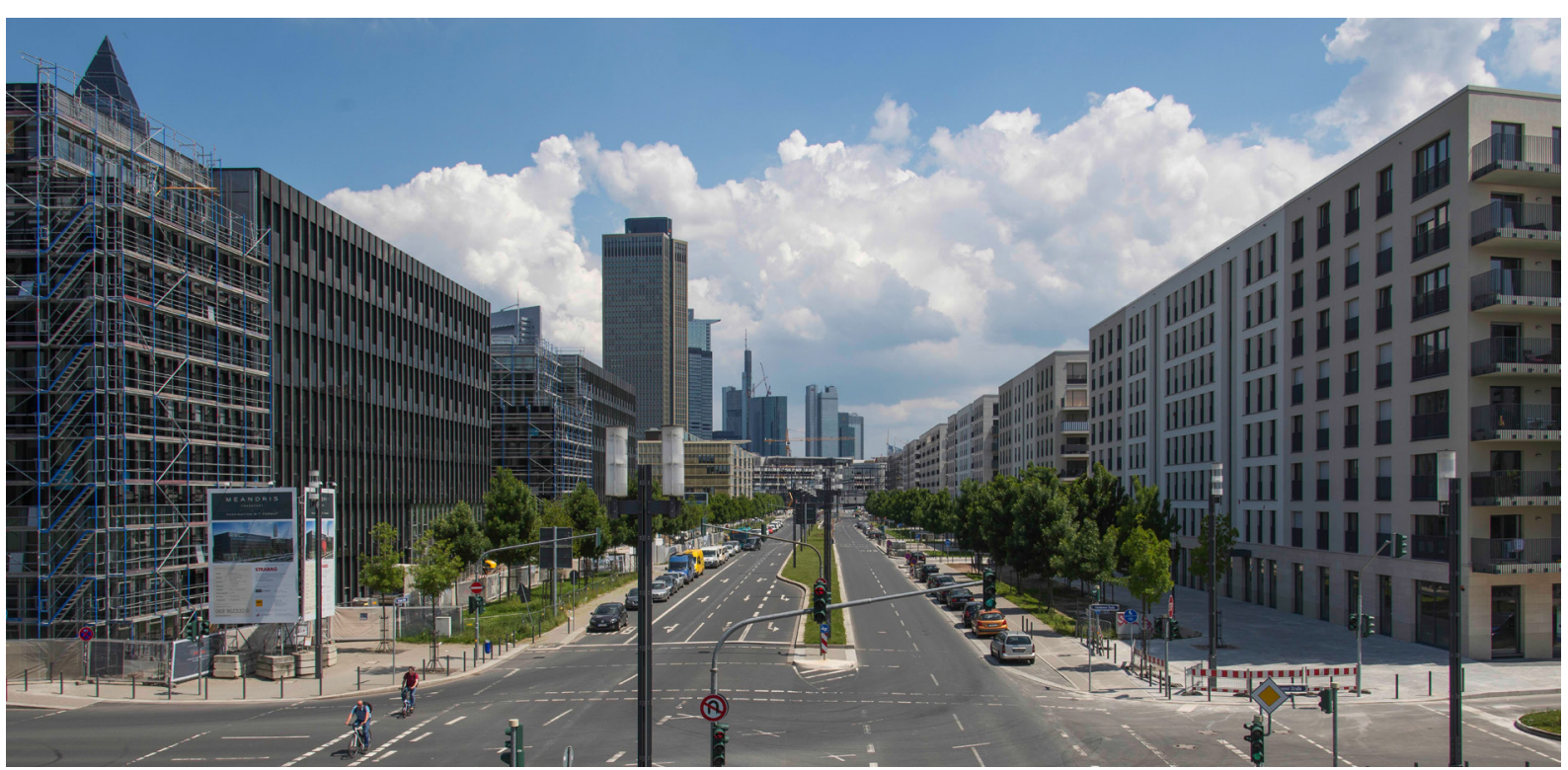


Kongresszentrum gebaut und 2013 beziehungsweise 2014 eröffnet. Darüber hinaus laufen gegenwärtig die konkreten Planungen für einen Teil jener Büro- und Wohnhochhäuser, die seit dem vorherigen Immobilienboom um die Jahrtausendwende im Hochhausrahmenplan der Stadt Frankfurt vorgesehen sind, von denen bisher aber nur das 200 Meter hohe, 2012 fertig gestellte Bürogebäude Tower 185 realisiert werden konnte. Demnach sollen an diesem Standort unter anderem ein zusätzliches Bürohochhaus, das mit 160 Metern höchste Wohngebäude Deutschlands sowie zwei bis drei weitere Wohnhochhäuser entstehen. Schließlich besteht nach wie vor Baurecht für den sogenannten Millenium-Tower, der mit bis zu 369 Meter einer der höchsten Wolkenkratzer Europas werden könnte, dessen Realisierung bislang aber höchst ungewiss ist.

Im Unterschied zu dieser sehr verdichteten Bebauung und dem hohen Anteil gewerblicher Nutzung war der westliche, auf den ehemaligen Gleisanlagen des Güterbahnhofes gelegeneTeil des Europaviertels von Beginn an mehrheitlich als weitläufiges Wohnquartier vorgesehen. Obwohl der Planungsprozess unter stärkerer Beteiligung von Ortsbeirat und Bürgerinitiativen verlief als im östlichen Teil, konnten Grundstückseigentümerin und Investoren auch hier in wichtigen Fragen (z. B. die Größe des zentral gelegenen Parks, der Verlauf der U-Bahn-Verlängerung und die Aufteilung der hierfür anfallenden Kosten oder die Preisstruktur der angebotenen Wohnungen) ihre Interessen durchsetzen (Langhagen-Rohrbach 2003: $161 \mathrm{ff}$.). Da die Bahn das Areal noch bis Mitte der 2000er Jahre als Rangierfläche nutzte, aber auch, weil ein zu diesem Zeitpunkt kriselnder Immobilienmarkt zu geringe Renditeaussichten eröffnete (Kirst 2006: 58 f., 80 f.), wurde in diesem Teil des Europaviertels erst Ende des Jahrzehntes, das heißt mit Einsetzen des jüngsten (Wohn-)Immobilienbooms, tatsächlich mit dem Bau begonnen. Seither entstehen dort rund um einen sechs Hektar großen Park, den Europagarten, verschiedene Wohnquartiere sowie mit den beiden Boulevards Mitte und West zwei gemischt genutzte Abschnitte, in denen Büros, Nahversorger, Kitas usw. untergebracht werden. Dominiert wird das Bild dabei von Projekten wie der gehobenen Wohnanlage Central \& Park oder dem selbsternannten ,Edelquartier' Parkend, in denen Mieter_innen und Käufer_innen das Gefühl vermittelt werden soll, „weit weg vom Mittelmaß“ (Werbeslogan) zu sein (Abb. 7).[10] Nicht weit entfernt von Parkend, am westlichen Ende des Europaviertels entstehen die beiden Wohntürme Westside Tower und Axis, wo für bis zu 20 Euro/m² Miete beziehungsweise knapp 10.000 Euro/ $\mathrm{m}^{2}$ Kaufpreis mit einem ConciergeService oder einem eigenen Hundewaschplatz geworben wird. Kaum geringer fallen die Preise für die Wohnungen im Objekt Harmonie West sowie dem Wohnhochhaus Praedium aus, welches die landeseigene, öffentliche Wohnungsbaugesellschaft Nassauische Heimstätte über ihr Tochterunternehmen NH ProjektStadt GmbH realisiert (Abb. 8). Etwas günstiger, für untere Einkommensschichten jedoch ebenfalls unerschwinglich, sind die 12 bis 15 Euro $/ \mathrm{m}^{2}$, welche die städtische Wohnungsbaugesellschaft $A B G$ Holding für ihre Mietwohnungen verlangt. Als Sozialwohnungen dienen lediglich die 400 Wohneinheiten der Helenenhöfe, die am nordwestlichen Ausgang des Europaviertels von dem privaten Wohnungsbauunternehmen Sahle Wohnen errichtet und später für 5 bis 5,50 Euro $/ \mathrm{m}^{2}$ an anspruchsberechtigte 

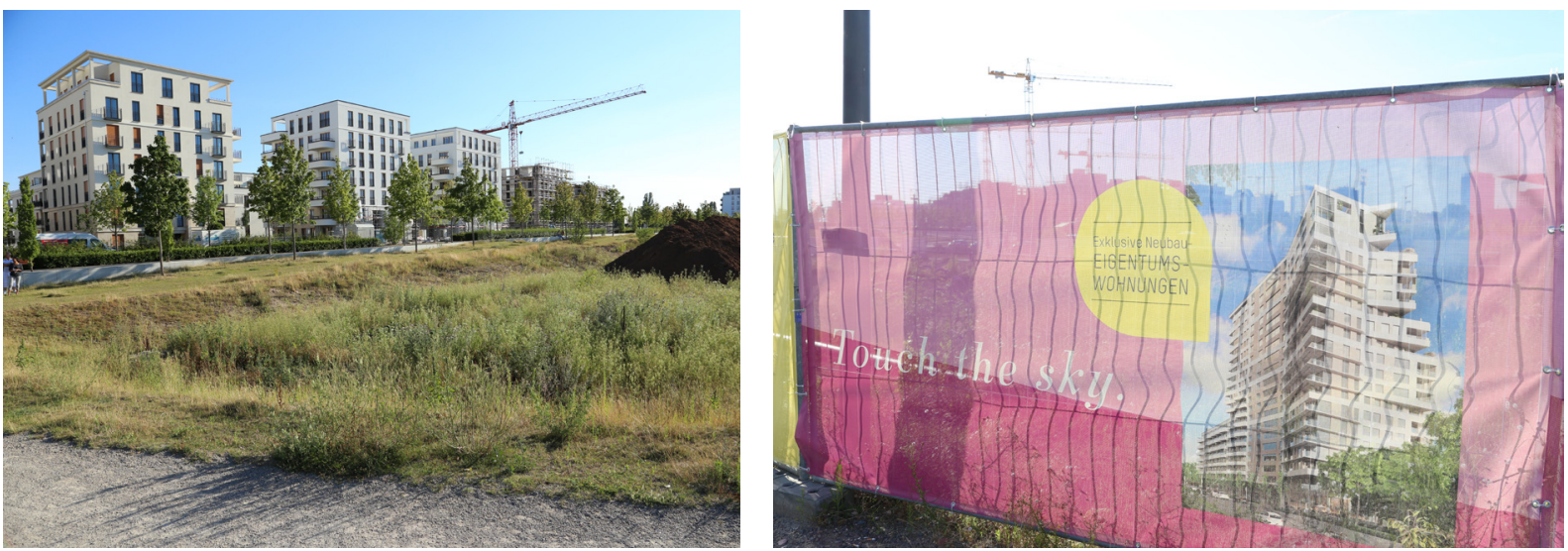

Abb. 7 Wohnblock Central \& Park im Europaviertel, Aug. 2014 (Foto: David Schommer)

Abb. 8 Baufeld für Praedium, ein Wohnhochhaus der Nassauischen Heimstätte, Aug. 2014 (Foto: David Schommer)
Haushalte vermietet werden. Vor dem Hintergrund, dass es sich dabei lediglich um knapp sieben Prozent des gesamten Wohnangebots handelt, mutet es beinahe zynisch an, wenn der städtische Planungsdezernent Olaf Cunitz (Die Grünen) die Helenenhöfe als deutlichen Beleg dafür wertet, „dass im Europaviertel nicht nur hochpreisige Wohnungen entstehen“ (FNP 08.03.2014). Zu berücksichtigen ist zudem, dass Sozialwohnungen in Hessen nur für fünf bis maximal 20 Jahre der Belegungs- und Preisbindung unterliegen. Nach Ablauf der Bindungsfrist dürfen aber auch diese Wohnungen zu Marktpreisen vermietet oder verkauft werden.

Als Fazit kann man festhalten, dass im Europaviertel nördlich des alten Gallus überwiegend gehobener und luxuriöser Wohnraum entsteht, weil politische Entscheidungsträger_innen bewusst darauf verzichtet haben, den potenziell tiefgreifenden Einfluss der öffentlichen Hand zu nutzen, um Wohnraum für unterschiedliche Einkommensgruppen zu ermöglichen. Stattdessen entsteht auf dem Areal des Europaviertels zu über 9o Prozent gehobenes Wohnen ( $\mathrm{ab} 12$ Euro $/ \mathrm{m}^{2}$ Miete), weil erstens die öffentlichen Flächen der DB profitorientiert verwertet und privatisiert worden sind, zweitens eine investorenfreundliche Stadtplanung weitgehend marktorientiert und „flexibel bis zum Anschlag“ (Heeg 2012) agiert hat, und drittens die städtischen (ABG) und landeseigenen (NH) Wohnungsbauunternehmen selbst profitorientiert handeln und lediglich hochpreisige Segmente bedienen.

\subsection{Wohnungsbau auf weiteren Konversionsflächen im alten Gallus}

Die dargestellten Entwicklungstendenzen beschränken sich indes nicht nur auf die Neubauten im Europaviertel, sondern treffen in leicht abgeschwächter Form ebenso auf die zahlreichen hochpreisigen Wohnungsbauprojekte zu, die infolge des kriseninduzierten Immobilienbooms seit etwa 2010 auf Konversionsflächen im Bereich des alten Gallus verwirklicht werden (Abb. 5). So entstehen etwa auf den wenigen noch vorhandenen Baulücken und Konversionsflächen im östlichen Bereich des Gallus ca. 300 Miet- und Eigentumswohnungen, die mit einer Preisspanne von 3.500 bis 5.500 Euro/m² zwar nicht ganz das Topniveau des benachbarten Europaviertels erreichen, sich aber ebenfalls eindeutig an ein zahlungskräftiges Klientel richten.

Noch umfangreicher fallen aktuell die Bauprojekte im westlichen Teil des Gallus aus, wo auf den Flächen ehemaliger Autohäuser, Handwerksbetriebe und Getränkemärkte sowie auf einem Areal der früheren Adlerwerke, das seit der Immobilienblase Anfang der 1990er Jahre brach lag, neue Miet- und 


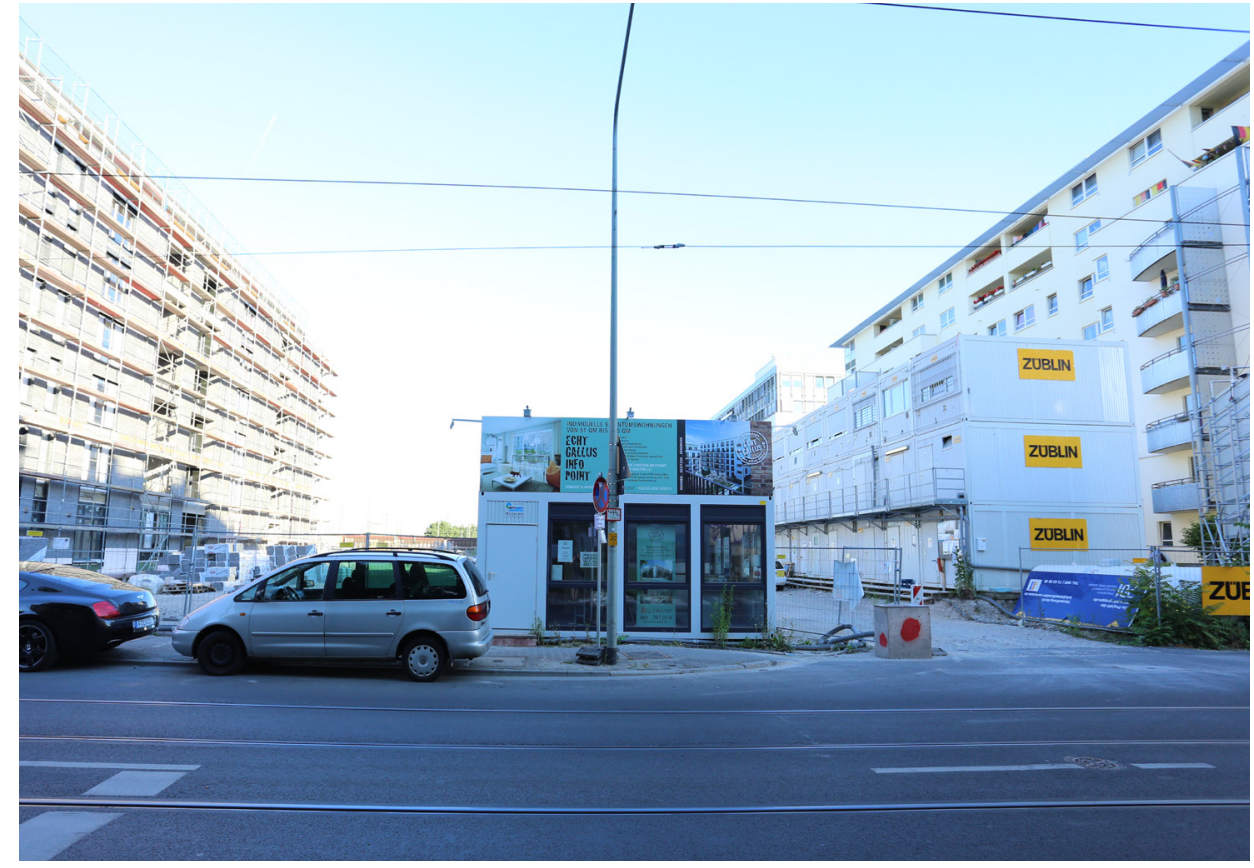

Eigentumswohnungen entstehen. Die Entwicklung konzentriert sich dabei räumlich zwischen Kleyerstraße und Frankenallee - allein hier werden bis zum Jahr 2016 verteilt auf mehrere Projekte über 2.00o neue Wohnungen errichtet. Wie umfassend der Aufwertungsprozess inzwischen ist, verdeutlicht die Preisstruktur der Neubauten: Während im Fall des bereits fertiggestellten ,Mehrgenerationenquartiers ${ }^{6}$ Frank an der Frankenallee zumindest ein geringer Teil der Wohnungen öffentlich gefördert ist und sich die Mieten (12 Euro $/ \mathrm{m}^{2}$ ) und Eigentumspreise (3.00o Euro/ $\mathrm{m}^{2}$ ) auch im frei finanzierten Bereich eher am unteren Durchschnitt des Europaviertels orientieren, fallen sie in den zum Teil frühzeitig an institutionelle Investoren verkauften Projekten wie den Lahn'schen Höfen oder den diversen Townund Roofhouses der Adler Quartiere deutlich höher aus (Abb. 9). Gleiches gilt für die 224 Apartments im Studio Eins oder für das von einem privaten Investor zum hochpreisigen ,Studentenwohnhaus“ umgebaute ehemalige Ordnungsamt, in dem die 390 maximal 24m² großen Zimmer um 550 Euro warm kosten sollen. Die generelle Stoßrichtung all dieser - und mehrerer weiterer - Projekte offenbart der Vorstandsvorsitzende des Immobilienunternehmens Opera One AG, Ralph Jerey, der angesichts des von seiner Firma geplanten „hoch attraktiven“ Wohnquartiers Gallus Gärten „von einem, neuen Gallusviertel ' [träumt] und von einer, Insel' spricht, auf der er mittleren und gehobenen Wohnungsbau realisieren will“(FAZ 29.07.2012). Einer solchen Entwicklung stehen schließlich auch die jüngst veröffentlichten Pläne für das Kleyerquartier nicht entgegen. Auf einer der letzten großen gewerblichen Konversionsflächen des Gallus am ehemaligen Standort der Firmen Avaya und Teves entstehen 1.200 Wohnungen, wovon 15 Prozent nach dem Frankfurter Mittelschichtsprogramm (15-35 Prozent unter der örtlichen Marktmiete) gefördert werden sowie weitere 15 Prozent auf Sozialwohnungen ( 5 Euro $/ \mathrm{m}^{2}$ ) entfallen sollen. Angesichts der sonstigen Entwicklung des Viertels erscheinen die 180 Sozialwohnungen, die auf diesem Weg maximal errichtet werden, jedoch bestenfalls als Tropfen auf den heißen Stein und kaum geeignet, der drohenden Verdrängung einkommensschwacher Schichten aus dem Gallus entgegenzuwirken.
Abb. 9 Adlerquartiere Louis am Park und Harry's Lofts, Kleyerstraße, Aug. 2014 (Foto: David Schommer) 
Abb. 10 Blick in die Lahnstraße im südlichen Gallus, Aug. 2014 (Foto: David Schommer)

Abb. 11 Blick in die Hattersheimerstraße an der Grenze zum Europaviertel, Aug. 2014 (Foto: David Schommer)

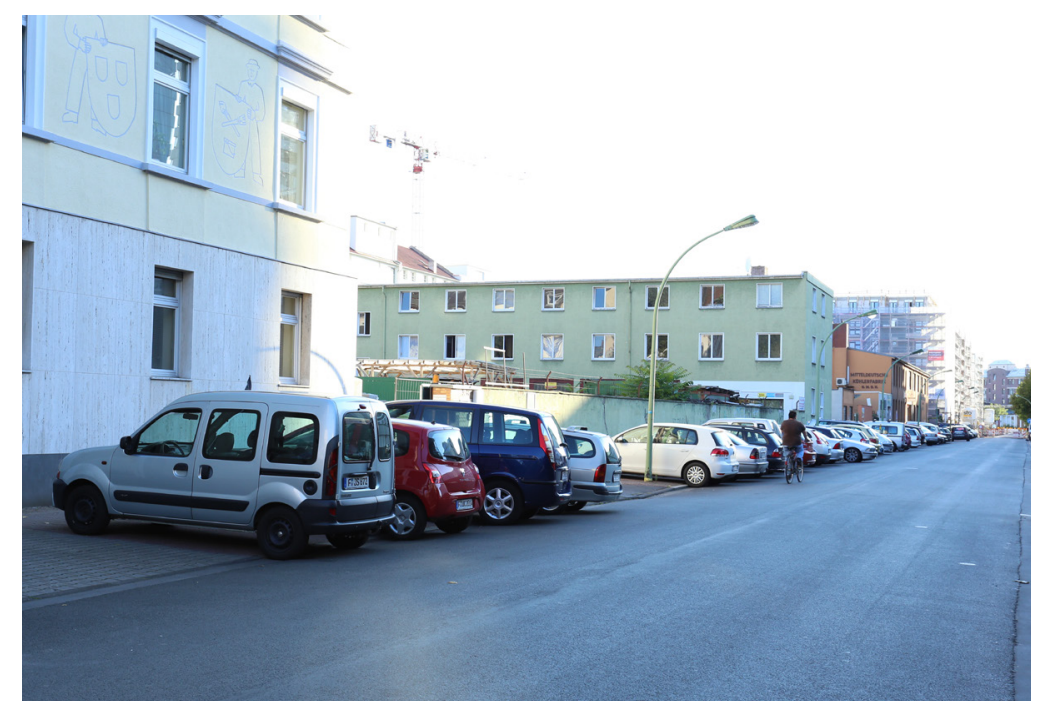
zeigen“ (Gutachterausschuss 2014: 11).

\section{3. ,Niemand hat die Absicht, das Gallus zu gentrifizieren...'}

Zusammenfassend hat also die Aussicht, im Gallus höhere Mieten und Wohnungspreise erzielen zu können, in den letzten Jahren eine umfassende Neubautätigkeit auf industriellen Brachflächen, insbesondere im Europaviertel, nach sich gezogen. Damit einher gehen Befürchtungen innerhalb der ortsansässigen Bevölkerung, dass das massive Investitionsvolumen im Neubau zu Ausstrahlungseffekten auf die noch relativ günstigen Bestandswohnungen in der unmittelbaren Nachbarschaft (Abb. 10 und 11) führen könnte und somit zukünftig eine Welle der Neubau-Gentrifizierung zu erwarten sei. Ein derartiger Zusammenhang wird jedoch bemerkenswerterweise von stadtpolitischen Entscheidungsträger_innen in der Regel vehement bestritten. So betont etwa der grüne Stadtplanungsdezernent Olaf Cunitz in einem Zeitungsinterview, dass der Wohnungsneubau im gehobenen Preissegment „eine sozialverträgliche Durchmischung und Aufwertung“ verspräche, wobei Sorgen von Anwohner_innen, das Wohnen im Gallus werde durch solche Projekte auf lange Sicht teurer, „unbegründet“ (FR 10.07.2013) seien. Vielmehr entstünde auf ehemaligen Gewerbebrachen „viel neuer Wohnraum ohne Verdrängung alteingesessener Mieter“ (FR 20.11.2013). Im Unterschied zum obersten Stadtplaner kommt der Gutachterausschuss für Immobilienwerte der Stadt Frankfurt hingegen zu einem etwas differenzierteren Fazit. In seinem Bericht von 2014 heißt es etwa, dass „der Wohnungsneubaumarkt und die Umwandlungswelle mittlerweile das Gallus erreicht [haben]. Neben der nachgefragten Innenstadtlage kommt die Nähe zum Europaviertel diesem Quartier zu Gute [Anm. d. Verf.: Natürlich kommt diese Entwicklung nicht, dem Quartier', sondern den dortigen Immobilienbesitzer_innen zu Gute]. Ob dies im Endeffekt zu einer Veränderung der Bevölkerungsstruktur führt, wird sich in den nächsten Jahren

Warum die Nähe zum Europaviertel den Immobilienbesitzer_innen womöglich zu Gute kommen wird und einen verschärften Prozess der NeubauGentrifizierung auslösen könnte, veranschaulichen die kleinräumigen Preisdifferenzen beziehungsweise Ertragslücken zwischen den beschriebenen Neubauarealen und dem alten Wohnungsbestand im Gallus. Gemäß dem IHK Wohnungsmarktbericht (2013: 15 f., eigene Berechnungen) liegen die

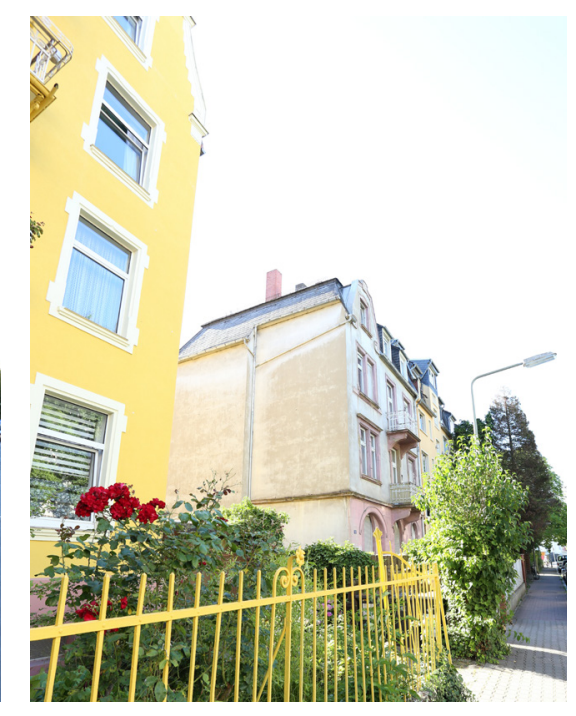


Kaltmieten bei Neuvertragsabschlüssen im Europaviertel um 40 Prozent und die Eigentumspreise um 70 Prozent über denjenigen im alten Gallus. Im Gegensatz zu den Beschwichtigungen des Frankfurter Stadtplanungsdezernenten deuten die erheblichen nahräumlichen Ertragslücken darauf hin, dass die geäußerten Befürchtungen vor einem Prozess der NeubauGentrifizierung sehr wohl berechtigt sind. Im Sinne der rent-gap-Theorie kann das gegenwärtig erzielte Mietniveau im Europaviertel aufgrund der gleichen Lagequalitäten zugleich als Annäherung an die potenzielle Grundrente im gesamten Gallus gelesen werden. Denn aus Sicht privater Investoren gibt es keinen Grund, warum nach Aufwertung, Modernisierung oder Abriss die Mieten im jetzigen Bestand dauerhaft niedriger sein sollen als diejenigen, die sich auf den Neubauflächen nebenan realisieren lassen.

Insofern Investoren also angesichts der zu erwartenden Ausstrahlungseffekte darauf spekulieren, zukünftig im Gallus ein höheres Mietniveau durchsetzen zu können, lassen sich erstens die daraus resultierenden immobilienwirtschaftlichen Strategien der Inwertsetzung als zentrale Dynamik einer Neubau-Gentrifizierung identifizieren. Diese wird zweitens durch die Funktionsweise des Mietspiegels befördert: Da die hohen Mietabschlüsse im Europaviertel und in den Neubauten im alten Gallus zukünftig in die Berechnung der ortsüblichen Vergleichsmiete einfließen[11], werden erhebliche Mieterhöhungen im Bestand ermöglicht - ein Zusammenhang, der anhand des im Mietspiegel 2014 neu eingeführten Innenstadtzuschlags für das östliche Gallus bereits sichtbar wird (vgl. Kapitel 2). Und schließlich ist drittens zu erwarten und zum Teil bereits zu beobachten, dass es infolge städtebaulicher Maßnahmen (wie jener des Programms Soziale Stadt) und des vermehrten Zuzugs einkommensstarker Haushalte zu einer symbolischen Aufwertung des städtischen Raums, zu neuen Nachfragemustern sowie zu einer veränderten Gewerbestruktur kommt, wodurch Verdrängungsprozesse verstärkt werden (Stabrowski 2014).

Vor diesem Hintergrund einer drohenden Neubau-Gentrifizierung ist es daher entscheidend, inwiefern die Stadt Frankfurt ihre wohnungspolitischen Instrumente - und hier insbesondere die kommunale Wohnungsbaugesellschaft ABG - nutzt, um zukünftig zumindest Verdrängungsprozesse im Bestand zu bremsen oder gar zu verhindern.

\section{Wohnen im Bestand: \\ Zur Rolle der städtischen Wohnungsbaugesellschaft $A B G$ Holding in gegenwärtigen Gentrifizierungsprozessen}

Die ABG Frankfurt-Holding ist ein 1991 gegründetes Tochterunternehmen der Stadt, das seitdem als Konzernmutter sämtlicher städtischer Wohnungsbaugesellschaften fungiert. Ende 2013 gehörten zum Bestand der ABG 50.483 Wohnungen, wovon 33.841 frei finanziert sind (ABG 2014a). Im Verhältnis zum gesamten Frankfurter Wohnungsangebot von 366.00o Einheiten entspricht dies knapp 14 Prozent aller Wohnungen (ABG 2013: 22) beziehungsweise gut 18 Prozent aller Mietwohnungen (eigene Berechnungen). Kontrolliert wird die als GmbH organisierte ABG von einem Aufsichtsrat, dem der Oberbürgermeister - derzeit Peter Feldmann (SPD) - vorsteht und der sich ansonsten je zur Hälfte aus Mitarbeiter_innen der ABG 
und Vertreter_innen der Stadt zusammensetzt (Stand 2014: 6x CDU, 3x Die Grünen, 1x SPD) (ABG 2014b: 68). In den Jahren 2012 und 2011 hat die ABG einen Konzernjahresüberschuss von jeweils rund 50 Millionen Euro erzielt, 2013 und 2014 ist diese Summe auf über 6o Millionen Euro angestiegen (ABG 2014a; 2013: 2). Von den Überschüssen wird jährlich ein einstelliger Millionenbetrag an die Stadt Frankfurt als einzige Gesellschafterin ausgezahlt. Da der überwiegende Anteil der Gewinne somit im Unternehmen verbleibt, ist die Eigenkapitalquote der Holding kontinuierlich von 18,8 Prozent (2001) auf 34,9 Prozent (2012) gestiegen (ABG 2013: 2; 2004: 2).

Während die ABG über ihren umfangreichen Bestand bereits einen erheblichen Einfluss auf gesamtstädtische Wohnungsmarktentwicklungen ausübt, kann die Stellung der ABG im Gallus sogar als nahezu marktbeherrschend charakterisiert werden. Von den ca. 16.00o Bestandswohnungen im Viertel (davon 1.800 in Sozialbindung) befinden sich 5.500 Wohneinheiten (davon 820 in Sozialbindung) im Besitz der stadteigenen Wohnungsbaugesellschaft (FAZ 29.07.2012). Die Bewirtschaftungspraxis des Wohnungskonzerns bestimmt somit wesentlich die Entwicklung des allgemeinen Mietniveaus im Stadtteil. Anders ausgedrückt könnte die ABG angesichts der Aufwertungsprozesse und der drohenden Neubau-Gentrifizierung direkt über die Mietentwicklung in den eigenen Beständen sowie indirekt über deren Eingang in die Berechnung der ortsüblichen Vergleichsmiete signifikant auf das Mietpreisniveau einwirken. Folglich entscheiden nicht anonyme Marktkräfte, sondern die Stadt vermittelt über ihr Wohnungsunternehmen darüber, ob einkommensschwache Haushalte aus ABG-Wohnungen verdrängt werden oder ob der stadteigene Wohnungsbestand dazu genutzt wird, insgesamt dämpfend auf die Mieten einzuwirken.

Bezüglich der Frage, inwiefern die Stadt ihren Handlungsspielraum nutzt, kommen Vertreter_innen und Kritiker_innen der ABG zu gänzlich unterschiedlichen Einschätzungen. So heben Vertreter_innen der ABG Holding sowie die mit ihr verbundenen städtischen Entscheidungsträger_innen stets hervor, dass die Holding auch einem sozialpolitischen Anspruch verpflichtet sei. Der ABG-Geschäftsführer Frank Junker betont etwa: „Vom Sozialhilfeempfänger bis zum Generaldirektor soll und kann jeder bei der ABG Wohnraum finden. [...] Damit sind wir gerade in Quartieren wie dem Gallus ein Garant dafür, dass es dort nicht zu Gentrifizierung kommt"(FR 24.02.2014). Als Begründung wird dabei angeführt, dass die ABG erstens nicht aus dem Bestand privatisiere, zweitens die Miete bei bestehenden Mietverträgen maximal bis zur ortsüblichen Vergleichsmiete anhebe (womit sie jedoch lediglich das deutsche Mietrecht respektiert) und drittens die Kappungsgrenze für Mieterhöhungen im Bestand fünf Prozentpunkte unter der gesetzlich vorgeschriebenen Grenze von 20 Prozent beziehungsweise jetzt 15 Prozent in drei Jahren liege (wodurch letztlich nicht die absolute Höhe der Miete, sondern nur die Geschwindigkeit der Mietsteigerung etwas stärker gedämpft wird).

Kritiker_innen halten dem entgegen, dass die ABG in den meisten Fällen ihre rechtlichen Möglichkeiten für Mietsteigerungen bis zur gesetzlichen Höchstgrenze ausschöpfe (FNP 16.01.2013) und sich daher in der Bewirtschaftungspraxis kaum von einem profitorientierten privaten Investor unterscheide. Dass diese Einschätzung der Realität durchaus näher kommt als die Eigendarstellung der ABG, verdeutlicht eine Reihe von Indizien. 
Erstens begreift sich die ABG selbst in erster Linie als „ein Wirtschaftsunternehmen“, das unter der Maxime agiert, nur „etwas [zu] machen“, wenn „wir damit Geld verdienen“ (Geschäftsführer Frank Junker zit. n. FR 24.02.2014). Demzufolge wird die ABG sozialpolitisch nur dann aktiv, wenn sie - ähnlich wie private Investoren auch - öffentliche Subventionen durch den Verkauf von Belegungsrechten oder im Form von zinsgünstigen Darlehen für geförderten Wohnraum in Anspruch nehmen kann. Aufgrund der Dominanz einer gewinnorientierten Grundausrichtung hat die Holding im Unterschied zu gemeinnützigen Wohnungsunternehmen kein intrinsisches Interesse daran, eigene finanzielle Ressourcen und Überschüsse für sozialpolitische Zwecke einzusetzen (Kuschinski 2014, Tharun/Körner 2001: 187ff.).

Zweitens liegen sowohl die Bestands- als auch die Wieder- und Neuvermietungsmieten der ABG nur jeweils leicht unter dem allgemeinen Durchschnittsniveau. Während etwa die Mietangebote bei privaten Vermietern im Gallus zwischen 13-16 Euro/m² schwanken, bietet die ABG auf ihrem Portal Wohnungen für 11,50-15 Euro/ $\mathrm{m}^{2}$ an und erzielt im freifinanzierten Neubau laut Angabe des Magistrats (Magistrat der Stadt Frankfurt 2014b) stadtweit einen durchschnittlichen Mietpreis von 12,06 Euro/m². Im Bestand lagen die ABG-Mieten Ende 2013 im Durchschnitt bei 7,64 Euro/m², also mit 5,4 Prozent nur leicht unter dem zu diesem Zeitpunkt gültigen Niveau des Mietspiegels von 8,08 Euro/m² (ebd.). Da der Mietspiegel aufgrund der spezifischen Erhebungsmethoden immer etwas über dem realen Durchschnitt liegt (Schardt 2012), kann man schlussfolgern, dass die Mieten in ABG Wohnungen sowohl bei Neuvermietung als auch im Bestand aller Voraussicht nach ungefähr dem jeweiligen Marktniveau entsprechen und damit (fast) genauso hoch sind wie bei privaten Eigentümern auch. Zu vermuten ist zudem, dass die geringfügigen Differenzen zu privaten Anbietern nicht aus dem sozialen Anspruch des Unternehmens resultieren, sondern vielmehr dem architektonisch weniger attraktiven Wohnungsbestand geschuldet sind. Denn im Vergleich zu privaten Eigentümern besitzt die ABG im Gallus überproportional viele einfache Wohnungen, die ab den 1920er Jahren im Rahmen des Wohnungsbauprogramms des Neuen Frankfurts errichtet worden sind - wie beispielsweise die Hellerhofsiedlung mit allein 1.200 Wohnungen (Abb. 12).

Drittens gab es laut Angabe des Magistrats der Stadt Frankfurt (2014a; 2013) im Bereich des Ortsbeirats 1 (wozu neben dem Gallus auch das Gutleutviertel, das Bahnhofsviertel und die Altstadt zählen) zwischen 2011 und 2013 insgesamt 266Zwangsräumungen. Dass davon 160 von öffentlichen Wohnungsunternehmen, das heißt überwiegend von der ABG, und nur 106 von privaten Eigentümern eingeleitet worden sind, spricht nicht unbedingt dafür, dass das städtische Wohnungsunternehmen gemäß einem sozialen Auftrag agiert. Schließlich sei viertens angemerkt, dass die Stadt Frankfurt 1999 eine nicht bindende Vereinbarung (den so genannten ,Frankfurter Vertrag') mit der ABG geschlossen hat, die vorsah, dass Wohnungen, die aus der Sozialbindung fallen, weiterhin zu Sozialmieten an berechtigte Einkommensgruppen vermietet werden. Der Vertrag gilt jedoch seit langem als gescheitert, da sich die städtische Wohnungsbaugesellschaft nicht an die Vereinbarung gehalten hat (Kuschinski 2014: 35). Dementsprechend kommt Eva Kuschinski (2014) in ihrer Studie zur Wohnungspolitik der ABG 
Abb. 12 Einfache Wohnungen in der Hellerhofsiedlung, Idsteiner Straße, Aug. 2014 (Foto: David Schommer)

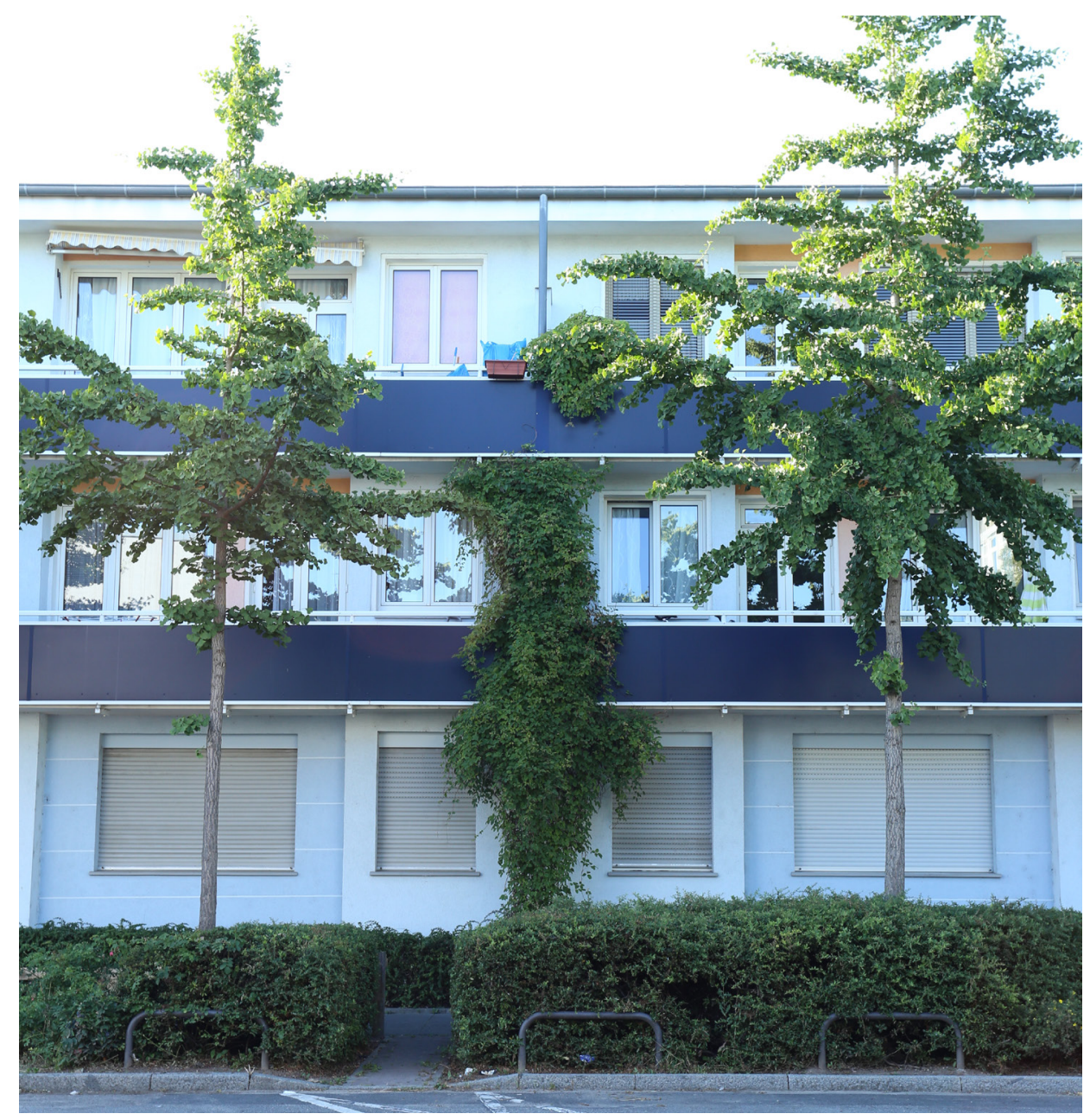

zu dem Schluss, dass die Holding aufgrund ihrer renditeorientierten internen Struktur und der damit verbundenen betriebswirtschaftlichen Ausrichtung kaum aus eigener Motivation dem sozialen Auftrag nachkommt, bezahlbaren Wohnraum für einkommensschwache Schichten sicherzustellen (vgl. auch Tharun/Körner 2001: 187 ff.). In einem solchen Sinne agiert die ABG nur dann, wenn sie explizit von der Stadt Frankfurt durch klare Vorgaben dazu gezwungen wird, wie es etwa bei der Absenkung der Kappungsgrenze geschehen ist, oder Fördermittel für Belegungsrechte beziehungsweise sozialen Wohnungsbau bereitgestellt werden.

Aus diesem Grund ist es entscheidend, die wohnungspolitische Ausrichtung der städtischen Politik in den Blick zu nehmen und zu fragen, auf welche politischen Ziele die Stadt Frankfurt ihre ABG verpflichtet. Angesichts einer sich seit den 1980er Jahren sukzessive formierenden Hegemonie der unternehmerischen Stadt (Schipper 2013a) ist diesbezüglich zu konstatieren, dass auch die wohnungspolitische Ausrichtung der Stadt Frankfurt spätestens seit Mitte der 1990er Jahre primär darauf abzielt, die Wettbewerbsfähigkeit der Stadt in der interurbanen Konkurrenz um einkommensstarke Haushalte der Mittel- und Oberschicht zu stärken und „Wissensnomaden für mindestens eine Generation an diesen Standort zu binden “(Oberbürgermeisterin Petra Roth 2010, zit. n. Schipper 2013a: 321). Diese politische Stoßrichtung spiegelt sich etwa stadtplanerisch in zahlreichen gehobenen Neubau-Projekten (Schipper 2013b) und wohnungspolitisch in der Halbierung des Bestands an öffentlich geförderten Sozialwohnungen von knapp 68.00o (1990) auf nun unter 30.00o Wohneinheiten wider (Schipper 2013a: 350). Die dem 
zugrunde liegende neoliberale Rationalität städtischen Regierens kennzeichnet ebenso die wohnungspolitischen Leitlinien der Stadt, die 2006 von der Stadtverordnetenversammlung beschlossen und zuletzt 2008 aktualisiert worden sind. Dort heißt es etwa, dass für die Verbesserung der sozialen Vielfalt stadtplanerisch „ein ausreichendes Wohnungsangebot für den mittleren bis gehobenen Bedarf" geschaffen und bei der Bereitstellung von Wohnbauflächen „die gehobenen Ansprüche verstärkt berücksichtigt“ (Stadt Frankfurt 2008: 7) werden müssten. Für den entsprechenden Neubau könnte auch die städtische Wohnungsbaugesellschaft als Investor gewonnen werden. Entgegen deren ursprünglichem sozialpolitischem Auftrag, primär in bezahlbaren Wohnraum für niedrige Einkommensschichten zu investieren, werden die in der $A B G$ Holding zusammengefassten kommunalen Wohnungsunternehmen nun also als „wichtige Partner und effektive Instrumente“ in die Pflicht genommen, „die Attraktivität der Stadt Frankfurt am Main als Wohn- und Wirtschaftsstandort zu erhalten“ (ebd.: 15 f.).

Betrachtet man also zusammenfassend das Selbstverständnis der ABG und die politischen Zwecke, die die Stadt Frankfurt mit ihrer Wohnungsbaugesellschaft verfolgt, so kann geschlussfolgert werden, dass die ABG Gentrifizierungsprozesse im Gallus (und anderswo) tendenziell eher befördert als bremst. Aufgrund ihrer binnenprivatisierten Struktur und der dominanten betriebswirtschaftlichen Rationalität begreift sich die ABG selbst als ,normales' Wohnungsunternehmen, welches primär gewinnorientiert zu wirtschaften hat. Um Gentrifizierung zu bremsen oder gar zu verhindern, müsste daher die Stadt als alleinige Gesellschafterin klare politische Vorgaben zur Deckelung von Mietpreisentwicklungen sowie zur Begrenzung von Renditeinteressen erlassen, die die Gemeinnützigkeit des Unternehmens verbindlich festschreiben. Zumindest bislang sind die wohnungspolitischen Leitlinien und Vorgaben aber eher durch gegenteilige Anweisungen gekennzeichnet, da die ABG per politischem Beschluss auf Gewinnorientierung getrimmt und bislang als Instrument der Aufwertung im interkommunalen Wettbewerb um gehobene Mittelschichten genutzt worden ist. Die wenigen materiellen Zugeständnisse, wie etwa die leicht abgesenkte Kappungsgrenze, sind im Verhältnis zu den faktischen Möglichkeiten eines Unternehmens, das jährlich über 60 Millionen Euro Gewinn erwirtschaftet, als marginal zu interpretieren.

\section{Fazit und Ausblick}

Die gegenwärtigen Verdrängungsprozesse im Gallus aus dem spezifischen Zusammenspiel von stadtpolitischen Aufwertungs- sowie immobilienwirtschaftlichen Verwertungsstrategien zu erklären, war Anspruch dieses Beitrages. Aufgezeigt haben wir dabei, dass eine innerstädtische rent gap als Motor von Gentrifizierungsprozessen interpretiert werden kann (Kapitel 2) und dass stadtpolitische Aufwertungsstrategien jahrzehntelang den Anstieg der potenziellen Grundrente ermöglicht und aktiv gefördert haben (Kapitel 3). Deutlich geworden ist ebenso, dass eine zentrale Lage in Verbindung mit stadtpolitischen Aufwertungsstrategien allein aber noch nicht notwendigerweise Gentrifizierungsprozesse induziert, sondern dies von ökonomischen Krisen- und Wachstumsphasen abhängt. Konkret haben im Falle des Gallus 
die stockende global-city-Formierung Frankfurts und der damit verknüpfte Einbruch am Immobilienmarkt Anfang der 1990er Jahre die sich bereits andeutenden Aufwertungstendenzen abrupt beendet. Erst die globale Finanzkrise von 2008 hat bewirkt, dass Kapital auf der Suche nach vermeintlich sicheren und profitablen Anlagesphären wieder verstärkt in (innerstädtische) Wohnungsmärkte wie das Gallus strömt, wodurch nun eine Welle der Neubau-Gentrifizierung angetrieben wird (Kapitel 4). Nichtsdestotrotz ist aber auch damit noch kein Automatismus der Verdrängung in Gang gesetzt, da die Stadt Frankfurt über ihre stadtplanerischen Instrumente (Bebauungspläne, städtebauliche Verträge) und vor allem mittels des stadteigenen Wohnungsunternehmens sowohl im Neubau eine soziale Mischung hätte durchsetzen können als auch einkommensschwache Mieter_innen im Bestand effektiv vor Verdrängung schützen kann (Kapitel 5).

Nahezuidealtypisch kann man daher am Gallus zeigen, dass Gentrifizierung kein natürliches Resultat rein ökonomischer Marktkräfte ist, sondern wesentlich von lokalen politischen Kräfteverhältnissen geprägt wird. Bislang waren selbige durch eine jahrzehntelange hegemoniale Zustimmung zur unternehmerischen Stadt gekennzeichnet, insofern städtische Eliten nahezu konsensual die Aufwertung innerstädtischer Viertel als Instrument im Wettbewerb um gehobene Mittelschichten und globale Wissensnomaden begrüßt haben (Schipper 2013a). Angesichts der eskalierenden Widersprüche und zunehmenden Proteste lassen sich gegenwärtig jedoch zumindest im Bereich der Wohnungspolitik gewisse Risse identifizieren. So fordert beispielsweise der seit Juli 2012 amtierende Oberbürgermeister Peter Feldmann (SPD) seit Sommer 2014, die Mieten in ABG-Wohnungen für drei Jahre praktisch einzufrieren, um Verdrängungsprozesse zu bremsen - womit er sich jedoch bislang nicht gegen die schwarz-grünen Mehrheiten in Magistrat, Stadtverordnetenversammlung und Aufsichtsrat durchsetzen konnte (Stadtverordnetenversammlung 2014: 56 ff.). Nichtsdestotrotz bieten derartige Brüche innerhalb der politischen Eliten durchaus Potenzial, um über außerparlamentarischen Druck lokale Kräfteverhältnisse tatsächlich substanziell zu verschieben und gegebenenfalls eine postneoliberale Wohnungspolitik durchzusetzen. Gegenwärtig unternehmen vor allem verschiedene Mieterinitiativen, zum Teil gemeinsam mit stadtpolitischen Aktivist_innen aus dem linken Spektrum, verstärkt Schritte in diese Richtung, indem sie sich mit eigenen Forderungen aktiv in die Stadtpolitik einmischen und im Vorfeld der für 2016 anstehenden Kommunalwahlen Druck von unten erzeugen.[12] In einer gesellschaftlichen Situation, in der angesichts breiter Diskussionen über „Die neue Wohnungsfrage“ (Schönig 2013) ein temporärer Mietenstopp zumindest wieder ernsthaft diskutiert werden kann, lassen sich dabei sogar weitergehende Forderungen nach einer Dekommodifizierung des Wohnens und einer grundlegenden Demokratisierung der städtischen Wohnungsbaugesellschaft stark machen.[13] An Konzepten, wie Wohnraum dauerhaft aus den Marktlogiken herausgelöst und demokratisch in Selbstverwaltung organisiert werden könnte, um Gentrifizierung auch langfristig zu verhindern, mangelt es jedenfalls nicht (Holm 2013b). 


\section{Endnoten}

[1] Z. B. die 2013 entstandene Dokumentation „Gallus bleibt Gallus“ von Sabine Hoffmann, Jana Schlegel und Jörg Hein.

[2] Abstrakte Kategorien marxistischer Gesellschaftstheorie empirisch zu operationalisieren, ist stets mit einer gewissen Herausforderung verbunden. Dies gilt auch für die Rententheorie (Clark 1995, Schipper 2013b). Die hier gewählte räumliche Darstellung der Mietniveaus kann daher tatsächlich nur als grobe Annäherung dienen, um auf die Existenz einer rent gap hinzuweisen. Real umfassen Mietzahlungen sowohl die Verzinsung des investierten Baukapitals als auch die Grundrente für die Verwertung des Bodens.

[3] Freilich gibt es auch in diesen Viertel noch gewisse kleinräumige Nischen, die etwa dadurch entstehen, dass Kleineigentümer_innen aus diversen Gründen nicht profitmaximierend wirtschaften und daher die rechtlichen Mieterhöhungspotenziale nicht voll ausschöpfen.

[4] Bodenrichtwerte beschreiben durchschnittliche Lagewerte, die von städtischen Gutachterausschüssen aus aktuell abgeschlossenen Kaufverträgen, unter anderem zur steuerlichen Bewertung des Grundbesitzes, kalkuliert werden. Bodenrichtwerte eignen sich als Annäherung zur Berechnung der Grundrente im marxschen Sinne, da die Gutachterausschüsse bestrebt sind, den reinen Bodenpreis unter Absehung des investierten Baukapitals zu ermitteln. Um die lagespezifischen Veränderungen in den Blick nehmen zu könne, wurden nur solche Gebiete ausgewählt, bei denen sich weder die Nutzungsart (Wohnen, Mehrfamilienhaus) noch die Bebauungsdichte verändert hat. Für eine ausführlichere Darstellung der Methode siehe Schipper (2013b).

[5] Um die tatsächlichen Verdrängungsdynamiken auf Nachbarschaftsebene in den Blick nehmen zu können, wäre ein methodisches Repertoire anzuwenden, welches sowohl soziale Aufwertungsprozesse (d. h. Austausch der Bevölkerung) als auch überdurchschnittliche immobilienwirtschaftliche Ertragssteigerungen erfasst. Einen überzeugenden Vorschlag liefert diesbezüglich Andrej Holm (2014), der am Beispiel Berlins die zeitliche Entwicklung der sozialen Lage (Arbeitslosigkeit, Abhängigkeit von Existenzsicherung, Kinderarmut) und der Angebotsmieten auf der Ebene von LOR-Planungsräumen (LOR = Lebensweltlich orientierte Räume) analysiert, um Gentrifizierungsgebiete zu identifizieren.

[6] Das städtische, nicht-profitorientierte Stadtplanungsprogramm Neues Frankfurt unter Leitung von Oberbürgermeister Ludwig Landmann und dem Stadtbaurat Ernst May erlangte in den 1920er Jahren internationale Berühmtheit. Vor dem Hintergrund einer eklatanten Wohnungsnot und dem Leitgedanken ,Bauen für das Existenzminimum folgend entstanden in Frankfurt 12.00o einfache Wohneinheiten für Arbeiterhaushalte.

[7] Dass diese Beschreibung gerade auch für den Wohnungsbestand der sich im städtischen Besitz befindlichen Aktienbaugesellschaft für kleine Wohnungen (ABG) und Hellerhof $A G$ zutraf, war - neben dem unmittelbaren Auslöser einer bis zu 60 prozentigen Mieterhöhung - eine der zentralen Ursachen für einen umfassenden Mietstreik, an dem sich im Jahr 1972/73 über 1.00o Mietparteien beteiligten. Das Gallus war dabei eines der Zentren dieser Auseinandersetzung (Häuserrat Frankfurt 1974: 213 ff.).

[8] Dieses Interesse ist selbst eine direkte Konsequenz der 1994 erfolgten Gründung der Deutschen Bahn AG als privatrechtliche, profitorientierte Aktiengesellschaft. Es fand seinen Ausdruck u. a. in dem Konzept Projekte 21, wonach in rund zwanzig deutschen (Groß-)Städten der Bahnbetrieb räumlich neu geordnet und die auf diesem Weg frei werdenden Betriebsflächen für groß angelegte Stadtentwicklungsprojekte genutzt werden sollten. In Gestalt von Frankfurt 21 war dieses Vorhaben auch in der Mainmetropole geplant. Während die Verlagerung des Hauptbahnhofes unter die Erde im Jahr 2001 aus Kostengründen (vorläufig) gescheitert ist, wurden die Pläne, das Areal des Hauptgüterbahnhofes als Stadtentwicklungsgebiet zu nutzen, ab 1997 als eigenständiges Projekt weiterverfolgt und schließlich in Gestalt des Europaviertels realisiert.

[9] Bei den beiden Grundstückseigentümerinnen handelte es sich zunächst um die Eisenbahnimmobilienmanagement GmbH (EIM), die ehemalige Bahn-Liegenschaften verkaufen und die Erlöse an den Bund abführen sollte, sowie um die Bahn-Tochter Deutsche Bahn Immobilien $\mathrm{GmbH}$ (DBImm), d. h. um zwei de facto öffentliche Unternehmen. EIM wurde 2001 in die Vivico Real Estate $\mathrm{GmbH}$ überführt und schließlich 2007 für über eine Milliarde Euro an die österreichische Immobilienfirma CA Immo verkauft, d. h. privatisiert. Ebenfalls 2007 verkaufte die Bahn ihre Anteile an der Aurelis Real Estate GmbH, 
die 2002/03 aus der DBImm hervorgegangen war, an ein Konsortium aus internationalen Investoren und Immobilienfonds. Entsprechend der Besitzverhältnisse zwischen diesen beiden Eigentümerinnen erfolgte die Entwicklung des Europaviertels getrennt zwischen einem kleineren, östlich der Emser Brücke gelegenen Teil (EIM/Vivico/CA Immo Deutschland) sowie einem größeren, westlichen Abschnitt (DBImm/Aurelis).

[10] Ein Gefühl, das unter gänzlich anderen Vorzeichen wohl auch die rumänischen Bauarbeiter im Europaviertel hatten, die im Oktober 2012 aus Protest gegen ihren Stundenlohn von 1,09 Euro ihre Arbeit niederlegten (FR 26.10.2012).

[11] Zur Funktionsweise von Mietspiegeln siehe Schardt (2012).

[12] Etwa im Rahmen des wohnungspolitischen Bündnisses „Eine Stadt für alle! Wem gehört die ABG?"; siehe http://www.stadt-fuer-alle.net/

[13] Siehe Forderungen des Bündnisses Eine Stadt für alle! (2015).

\section{Autor_innen}

Sebastian Schippers Forschungsschwerpunkte beinhalten Stadtpolitik, politische Ökonomie des Wohnens und städtische soziale Bewegungen.

s.schipper@geo.uni-frankfurt.de

Felix Wiegands Forschungsschwerpunkte beinhalten die urbane Dimension von Krisen, Austeritätspolitiken und sozialen Kämpfen sowie das Verhältnis von Stadt und Staatlichkeit. wiegand@em.uni-frankfurt.de

\section{Literatur}

ABG Frankfurt-Holding (2014a): ABG setzt Maßstäbe für Wohnungsversorgung. Pressemitteilung vom 09.07.2014. http://www.frankfurt.de (letzter Zugriff am 18.07.2014).

ABG Frankfurt-Holding (2014b): Geschäftsbericht 2013. http://www.abg-fh.com/images/ content/geschaeftsberichte/GB_2013.pdf (letzter Zugriff am 08.09.2014).

ABG Frankfurt-Holding (2013): Geschäftsbericht 2012. http://www.abg-fh.com/images/ content/geschaeftsberichte/ABG-GB-2012_web.pdf (letzter Zugriff am 08.09.2014).

ABG Frankfurt-Holding (2004): Geschäftsbericht 2003. http://www.abg-fh.com/images/ content/geschaeftsberichte/ABG-GB_2003.pdf (letzter Zugriff am 08.09.2014).

Amt für Wohnungswesen der Stadt Frankfurt am Main (2014): Mietspiegel 2014. Frankfurt am Main: Zarbock.

Amt für Wohnungswesen der Stadt Frankfurt am Main (2012): Mietspiegel 2012. Frankfurt am Main: Zarbock.

Becker, Helmut (1986): Wer klassenbewusst war, war im Konsum: Arbeiterkinder und -jugendliche im Gallus-Viertel 1918-1933. Frankfurt am Main: dipa.

Borris, Maria (1973): Ausländische Arbeiter in einer Großstadt. Eine empirische Untersuchung am Beispiel Frankfurt. Frankfurt am Main: Europäische Verlagsanstalt.

Brenner, Neil / Theodore, Nik (2002): Cities and the geographies of actually existing neoliberalism. In: Antipode 33/3, 349-379.

Bürgeramt, Statistik und Wahlen (2013): Materialien zur Stadtbeobachtung, Heft 15. http:// frankfurt.de/sixcms/media.php/678/MSB_ST_15_Kapitel_4.pdf (letzter Zugriff am 22.09.2014).

Bürgeramt, Statistik und Wahlen (2012): Materialien zur Stadtbeobachtung, Heft 13. http:// frankfurt.de/sixcms/media.php/678/MSB_ST_13_Kapitel_4.pdf (letzter Zugriff am 22.09.2014).

Clark, Eric (1995): The rent gap re-examined. In: Urban Studies 32/9, 1489-1503.

Davidson, Mark / Lees, Loretta (2005): New-build gentrification and London's riverside renaissance. In: Environment and Planning A 37/7, 1165-1190.

Eine Stadt für alle! (2015): Forderungen. http://www.autistici.org/abgkampagne/ wp-content/uploads/2015/o4/Forderungen.pdf (letzter Zugriff am 26.10.2015) 
Frankfurter Allgemeine Zeitung (29.07.2012): Das Gallusviertel steht vor dem Wandel. http://www.faz.net/aktuell/rhein-main/frankfurt-das-gallusviertel-steht-vor-demwandel-11836528.html (letzter Zugriff am 08.09.2014).

Frankfurter Neue Presse (16.01.2013): ABG setzt auf Energieeffizienz. http://www.fnp. de/lokales/frankfurt/ABG-setzt-auf-Energieeffizienz;art675,159771 (letzter Zugriff am 08.09.2014).

Frankfurter Neue Presse (o8.03.2014): Europaviertel wird sozial gemischt. http://www.fnp. de/lokales/frankfurt/Europaviertel-wird-sozial-gemischt;art675,776504 (letzter Zugriff am 31.10.2014).

Frankfurter Rundschau (15.09.2012): Gallus Gentrifizierung. Besser als sein Ruf.http://www. fr-online.de/frankfurt/gallus-gentrifizierung-besser-als-sein-ruf,1472798,17256776.html (letzter Zugriff am 15.06.2014).

Frankfurter Rundschau (26.10.2012): Frankfurt Neubaugebiet Europaviertel: 1,09 Stundenlohn. http://www.fr-online.de/frankfurt/frankfurt-neubaugebiet-europaviertel1-09-euro-stundenlohn,1472798,20722696.html (letzter Zugriff am 31.10.2014).

Frankfurter Rundschau (10.07.2013): Luxuswohnungen im Gallusviertel. http://www. fr-online.de/frankfurt/frankfurt-wohnungsmarkt-luxuswohnungen-im-gallusviertel,1472798,23656738.html (letzter Zugriff am 08.06.2014).

Frankfurter Rundschau (20.11.2013): Die Stadt muss dichter werden. Interview mit Olaf Cunitz. URL: http://www.fr-online.de/frankfurt/wohnen-in-frankfurt--die-stadt-mussdichter-werden-,1472798,25092998.html (letzter Zugriff am 05.09.2014).

Frankfurter Rundschau (24.02.2014): Jeder kann Wohnraum finden. Interview mit Frank Junker. http://www.fr-online.de/stadtentwicklung/wohnen-in-frankfurt--jeder-kannwohnraum-finden-,26042926,26327426.html (letzter Zugriff am 12.06.2014).

Gutachterausschuss für Immobilienwerte (2014): Immobilienmarktbericht für den Bereich Frankfurt am Main. http://www.frankfurt.de/sixcms/media.php/738/MB_2014HJ1.pdf (letzter Zugriff am 09.10.2015).

Harvey, David (2013): Rebellische Städte. Vom Recht auf Stadt zur urbanen Revolution. Berlin: Suhrkamp.

Häuserrat Frankfurt (Hg.) (1974): Wohnungskampf in Frankfurt. München: Trikont.

Heeg, Susanne (2013): Wohnungen als Finanzanlage. Auswirkungen von Responsibilisierung und Finanzialisierung im Bereich des Wohnens. In: sub\urban 1/1, 75-99.

Heeg, Susanne (2012): Flexibel bis zum Anschlag: Bauen und Planen für die Global City Frankfurt. In: AK Kritische Geographie Frankfurt (Hg.): Wem gehört Frankfurt? Dokumentation des aktionistischen Kongresses vom März 2012. Frankfurt am Main: Institut für Humangeographie, 75-85

Holm, Andrej (2013a): Berlin's gentrification mainstream. In: Matthias Bernt / Britta Grell / Andrej Holm (Hg.): The Berlin Reader. A Compendium on Urban Change and Activism. Bielefeld: transcript, 171-188.

Holm, Andrej (2013b): Wohnen als Soziale Infrastruktur. http://www.links-netz.de/pdf/T_ holm_wohnen.pdf (letzter Zugriff am 08.10.2014).

Holm, Andrej (2014): Gentrifizierung mittlerweile ein Mainstreamphänomen? In: Informationen zur Raumentwicklung 4, 277-290.

IHK (Industrie- und Handelskammer) (2013): Wohnungsmarktbericht für den IHK-Bezirk Frankfurt am Main 2013. http://www.frankfurt-main.ihk.de/cgi-bin/sitestat?t=/ imperia/md/content/pdf/standortpolitik/ immoblienmarktberichte/wohnungsmarktbericht_2013.pdf (letzter Zugriff am 18.07.2014).

Kirst, Volker (2006): Die Entwicklung des Gallusviertels seit 1970 unter besonderer Berücksichtigung des Strukturwandels an drei Beispielen. Frankfurt am Main: Goethe-Universität, Institut für Kulturgeographie, Stadt- und Regionalforschung. Unveröffentlichte Diplomarbeit.

Klagge, Britta (2005): Armut in westdeutschen Städten. Strukturen und Trends aus stadtteilorientierter Perspektive. Stuttgart: Franz Steiner.

Krätke, Stefan (2014): Cities in contemporary capitalism. In: International Journal of Urban and Regional Research 38/5, 1660-1677.

Kuschinski, Eva (2014): Sozialer Wohnungsbau lohnt sich nicht - Ökonomisierung der Frankfurter Wohnungspolitik am Beispiel der ABG Frankfurt Holding. In: Susanne Heeg / Marit Rosol (Hg.): Gebaute Umwelt. Aktuelle stadtpolitische Konflikte in Frankfurt am Main und Offenbach. Frankfurt am Main: Institut für Humangeographie, 23-40. 
Langhagen-Rohrbach, Christian (2003): Räumliche Planung in Deutschland und der Schweiz im Vergleich. Rahmenbedingungen, Akteure und praktische Umsetzung, dargestellt an den Beispielen Europaviertel (Frankfurt am Main) und Zürich West, Frankfurt am Main: Rhein-Mainische Forschung.

Lauer-Seidelmann, Irmgard (2012): Kamerun - das sind wir. Das Gallus - ein Frankfurter Stadtteil. Offenbach: Lindermann.

Lees, Loretta / Slater, Tom / Wyly, Elvin (2008): Gentrification. New York: Routledge.

Magistrat der Stadt Frankfurt (2013): Stellungnahme vom 03.06.2013, ST 780. http://www. stvv.frankfurt.de/download/ST_780_2013.pdf (letzter Zugriff am 08.09.2014).

Magistrat der Stadt Frankfurt (2014a): Stellungnahme vom 13.06.2014, ST 821. http:// www.stvv.frankfurt.de/download/ST_821_2014.pdf (letzter Zugriff am 08.09.2014).

Magistrat der Stadt Frankfurt (2014b): Frage vom 28.03.2014, F 1386. http://www.stvv. frankfurt.de/download/F_1386_2014.pdf (letzter Zugriff am 08.09.2014).

Marcuse, Peter (1986): Abandonment, gentrification, and displacement: The linkages in New York City. In: Neil Smith / Peter Williams (Hg.): Gentrification of the City. Boston: Allen \& Unwin, 153-177.

Ronneberger, Klaus / Keil, Roger (1995): Ausser Atem - Frankfurt nach der Postmoderne. In: Hansruedi Hitz / Ute Lehrer / Roger Keil (Hg.): Capitales Fatales: Urbanisierung und Politik in den Finanzmetropolen Frankfurt und Zürich. Zürich: Rotpunktverlag, 284-353.

Schardt, Jürgen (2012): Das bundesdeutsche Vergleichsmietensystem und der Frankfurter Mietspiegel 2010. Frankfurt am Main: Institut für Humangeographie.

Schipper, Sebastian (2013a): Genealogie und Gegenwart der unternehmerischen Stadt. Neoliberales Regieren in Frankfurt am Main, 1960 - 2010. Münster: Westfälisches Dampfboot.

Schipper, Sebastian (2013b): Global-City-Formierung, Gentrifizierung und Grundrentenbildung in Frankfurt am Main. In: Zeitschrift für Wirtschaftsgeographie 57/4, 185-200.

Schönig, Barbara (2013): Die neue Wohnungsfrage. In: Blätter für deutsche und internationale Politik 2, 17-20.

Smith, Neil (1996): The New Urban Frontier. Gentrification and the Revanchist City. London: Routledge.

Smith, Neil (2002): New Globalism, new Urbanism: gentrification as global urban strategy. In: Antipode 34/3, 427-450.

Stabrowski, Filip (2014): New-build gentrification and the everyday displacement of Polish immigrant tenants in Greenpoint, Brooklyn. In: Antipode 46/3, 794-815.

Stadt Frankfurt (2006): Soziale Stadt Gallusviertel: Integriertes Handlungskonzept. http:// www.stadtplanungsamt-frankfurt.de (letzter Zugriff am 31.10.2014).

Stadt Frankfurt (2008): Wohnungspolitische Leitlinien. http://www.frankfurt.de/sixcms/ media.php/738/o81219\%20Leitlinien_Fassung\%202008.pdf (letzter Zugriff am 08.07.2014).

Stadtverordnetenversammlung Frankfurt (2014): Wortprotokoll über die 32. Plenarsitzung. http://www.stvv.frankfurt.de/download/WOP_32_26-06-2014.pdf (letzter Zugriff am 08.10.2014).

Tharun, Elke / Körner, Wilfried (2001): Wandel und Kontinuität in der Wohnungspolitik Die Entwicklung des kommunalen und zentralstaatlichen Einflusses auf den Wohnungsmarkt Frankfurt am Main seit 1871. In: Klaus Wolf / Frank Schymik (Hg.): 75 Jahre Rhein-Mainische Forschung. 1925 - 2000. Frankfurt am Main: Universität Frankfurt IDG 162-194.

Tharun, Elke / Unterwerner, Petra (1993): Transformationsprozesse in innenstadtnahen Industrie- und Arbeitervierteln - das Beispiel des Frankfurter Gallusviertels. In: Niemz, Günter (Hg.): Geographische Lehrwanderungen und Exkursionen in Hessen und Nachbarräumen. Frankfurt am Main: Selbstverlag des Instituts für Didaktik der Geographie, 304-315.

Welz, Gisela (1992): Das Gallus. Deindustrialisierung und Tertiarisierung eines Frankfurter Stadtteils. In: Projektgruppe des Ludwig-Uhland-Instituts für Empirische Kulturwissenschaften (Hg.): Weltstadt Frankfurt am Main? Multikultur Journal. Tübingen: Universität Tübingen, 81-87.

WobiG (1998): Spaß muß sein. Das Urban Entertainment Center am Frankfurter Güterbahnhof. In: diskus. Frankfurter StudentInnen Zeitschrift, 1/98. http://www.copyriot. com/diskus/1_98/8.htm (letzter Zugriff am 08.10.2014). 


\section{New-build gentrification and the global financial crisis}

Since the beginning of the global financial crisis in 2008, many urban centers in Germany are faced with a new wave of gentrification. By focusing on the working class neighborhood Gallus in Frankfurt/ Main, the paper aims at explaining the recent displacement processes of low-income households by looking at both municipal-led urban renewal plans and real estate investment strategies. Thereby, we argue that the local political elites, deeply entrenched in neoliberal rationalities, have actively fostered gentrification processes and the development of a rent gap within this traditional working class neighborhood over decades - a rent gap that, however, has only gained its relevance in the aftermath of the global financial crisis. Therefore, the example of Gallus shows that gentrification and displacement are strongly dependent on local power relations; while the rhythm is, at the same time, dictated by real estate investment cycles. 